\title{
An Integrative Model of Plant Gravitropism Linking Statoliths Position and Auxin Transport
}

\author{
Nicolas Levernier*, Olivier Pouliquen and Yoël Forterre* \\ Aix Marseille University, CNRS, IUSTI, Marseille, France
}

Gravity is a major cue for the proper growth and development of plants. The response of plants to gravity implies starch-filled plastids, the statoliths, which sediments at the bottom of the gravisensing cells, the statocytes. Statoliths are assumed to modify the transport of the growth hormone, auxin, by acting on specific auxin transporters, PIN proteins. However, the complete gravitropic signaling pathway from the intracellular signal associated to statoliths to the plant bending is still not well-understood. In this article, we build on recent experimental results showing that statoliths do not act as gravitational force sensor, but as position sensor, to develop a bottom-up theory of plant gravitropism.

OPEN ACCESS

Edited by:

Christophe Godin, Institut National de Recherche en Informatique et en Automatique,

France

Reviewed by:

Eric M. Kramer,

Bard College at Simon's Rock,

United States

Eugenio Martín Azpeitia,

University of Zurich, Switzerland

*Correspondence:

Nicolas Levernier

nicolas.levernier@univ-amu.fr Yoël Forterre

yoel.forterre@univ-amu.fr

Specialty section:

This article was submitted to Plant Biophysics and Modeling, a section of the journal

Frontiers in Plant Science

Received: 11 February 2021 Accepted: 03 March 2021 Published: 29 March 2021

Citation:

Levernier $N$, Pouliquen $\mathrm{O}$ and Forterre Y (2021) An Integrative Model of Plant Gravitropism Linking Statoliths

Position and Auxin Transport.

Front. Plant Sci. 12:651928. doi: 10.3389/fpls.2021.651928
The main hypothesis of the model is that the presence of statoliths modifies PIN trafficking close to the cell membrane. This basic assumption, coupled with auxin transport and growth in an idealized tissue made of a one-dimensional array of cells, recovers several major features of the gravitropic response of plants. First, the model provides a new interpretation for the response of a plant to a steady stimulus, the so-called sine-law of plant gravitropism. Second, it predicts the existence of a gravity-independent memory process as observed recently in experiments studying the response to transient stimulus. The model suggests that the timescale of this process is associated to PIN turnover, calling for new experimental studies.

\section{Keywords: plant tropism, gravity sensing, auxin signaling, PIN trafficking, modeling}

\section{INTRODUCTION}

The detection of gravity by plants and the resulting growth response (gravitropism) offer a fascinating illustration of a multi-scale perception mechanism in living organisms (Figure 1) (Moulia and Fournier, 2009; Morita, 2010; Toyota and Gilroy, 2013). It originates in specific cells, called statocytes, where tiny starch-accumulating amyloplasts acting as statoliths sediment under gravity at the bottom of the cells (Figure 1C). When the plant is inclined, the repositioning of statoliths under gravity induces a relocalization of auxin transporters (PIN proteins) at the membrane of statocytes, which generates a lateral transport of auxin toward the lower side of the shoot or the root (Cholodni-Went hypothesis) (Figure 1B). In turn, this asymmetry in auxin concentration induces a differential growth across the plant organ, and thus its bending toward the gravity vector (Figure 1A). Since the pioneering works of the Darwins and Sachs on plant tropisms (Darwin and Darwin, 1880; Sachs, 1887), progress has been made on every step of this gravitropic signaling pathway. Yet, basic questions remain unanswered. In particular, it is still not clear how the first physical signal generated by the sedimentation of statoliths is converted into biochemical signals downstream, to eventually produce the growth response at the plant scale (Nakamura et al., 2019b). 


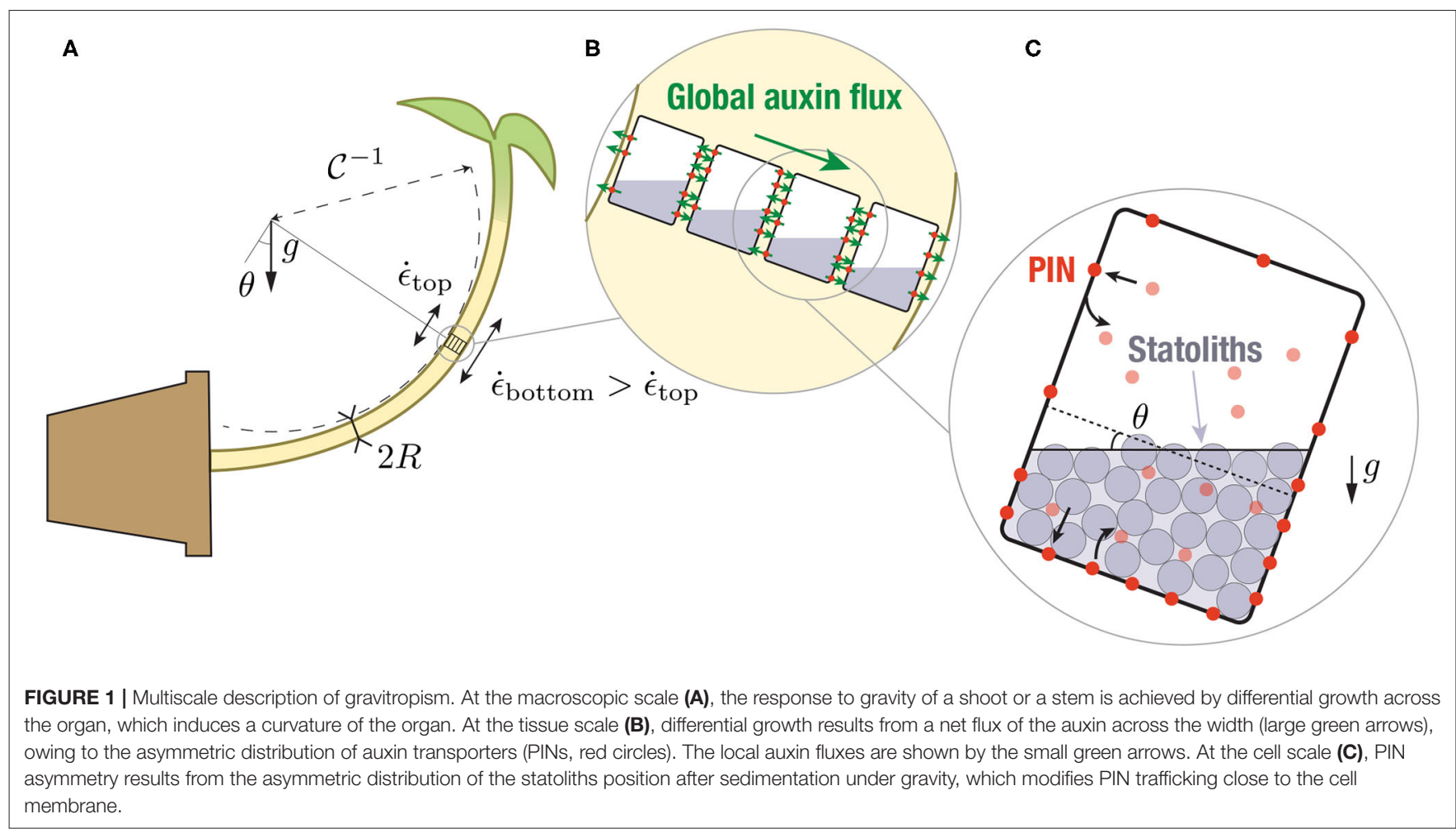

Recently, insights into the sensing mechanism and the transduction pathway have been obtained from experiments both at the macroscopic and microscopic levels. First, the gravitropic response to permanent stimuli (inclination of the plant), the socalled sine-law of gravitropism (Sachs, 1887; Larsen, 1969; Iino et al., 1996; Galland, 2002; Dumais, 2013), was found to depend on the inclination but, surprisingly, not on the intensity of gravity (Chauvet et al., 2016). Hence, statocytes behave like inclination sensors not force sensors as previously believed. An important consequence is that the initial gravity stimulus for gravitropism should be the position of the statoliths within statocytes (Pouliquen et al., 2017). This position-sensor hypothesis gained a mechanistic support from the direct observation of statoliths motion under gravity stimulation (Bérut et al., 2018). Unlike a pile of macroscopic grains like sand, statoliths were found to move and flow at any inclination. This liquid-like behavior comes from the random agitation of the statoliths, whose origin is not thermal but arises from the interaction of statoliths with the acto-myosin cytoskeleton inside the cell (Sack et al., 1986; Saito et al., 2005; Nakamura et al., 2011). A second insight came from dose-response like experiments on wheat coleoptiles, in which the gravity stimulus was applied during a transient period only (Chauvet et al., 2019). When the shoots were inclined for short period of time, the gravitropic response was found to deviate from the steady response and decay. The transition occurred for a time $\tau_{\text {memory }} \sim 15 \mathrm{~min}$, which was independent of gravity and much larger than the statoliths sedimentation time. This observation suggested the existence of a memory-integration process in the gravitropic signaling pathway, independent of the statoliths dynamics, which integrates the initial signal induced by statoliths displacement.

To account for these observations (position-sensor hypothesis, memory time independent of $g$ ), Chauvet et al. (2019) built a mathematical model of gravitropism in which the gravitropic signal controlling the differential growth was linked to the statoliths position by an integrative process of timescale $\tau_{\text {memory }}$ (a similar approach was used in Meroz et al., 2019). Once coupled to the statoliths dynamics and the tropic growth motion, the model was able to reproduce the transient gravitropic response observed experimentally. However, Chauvet et al. (2019)'s model was built on two ad-hoc postulates. First, it assumed that the relation between the gravitropic signal and the statoliths position is known and given by the sine-law. Second, it postulated the existence of the integrative process and time scale $\tau_{\text {memory, without explaining its origin. The spatio-temporal }}$ dynamics of the molecular processes acting between the statoliths and the growth response, such as the dynamics of PIN proteins and auxin transport, was not described.

The objective of this paper is to fill this gap by building an integrative model of plant gravitropism that bridges the different scales of the process: (i) the initial intracellular gravitropic signal encoded in the statoliths position, (ii) PINs dynamics at the cellular level, (iii) auxin transport at the tissue level and, finally, (iv) differential growth and curvature at the plant organ scale. Previous models of plant gravitropism mainly focused either on the macroscopic scale, describing how the complex spatiotemporal evolution of the organ shape results from the interplay between differential growth and the slender geometry of the 
organ (Bastien et al., 2013, 2014; Chelakkot and Mahadevan, 2017; Moulton et al., 2020), or on the tissue level, modeling growth mechanics (Dyson et al., 2014) and auxin transport (Band et al., 2012; Fendrych et al., 2018; Retzer et al., 2019) in realistic tissue geometries. In these latter models, the distribution of PINs in response to plant inclination was prescribed and not linked to the intracellular dynamics of the statoliths. This is precisely the goal of our study. Building on the recent position-sensor hypothesis, we propose a simple but generic model of interaction between statoliths and PINs trafficking at the cell membrane, that we couple with the classical equations of auxin transport and tissue growth. We then study the gravitropic response predicted by the model for steady and unsteady gravity stimuli, comparing the results with the experiments of Chauvet et al. $(2016,2019)$.

\section{MATERIALS AND METHODS}

\subsection{Link Between Gravitropic Curvature, Differential Growth, and Auxin Concentration Gradient}

At the plant scale, the gravitropic response is characterized by the curvature of the organ resulting from differential growth, which itself results from auxin gradients (Cholodny-Went hypothesis). The first step of the model is thus to relate those three quantities. For a slender organ like a shoot or a stem, the rate of change of the local curvature $\mathcal{C}$ is related to differential growth through the following kinematic relationship: $R \frac{\mathrm{d} \mathcal{C}}{\mathrm{d} t}=\frac{1}{\tau_{g}} \times \frac{\dot{\epsilon}_{\text {bottom }}-\dot{\epsilon}_{\text {top }}}{2 \dot{\epsilon}_{\text {mean }}}$, where $R$ is the radius of the organ, $\dot{\epsilon}_{\text {bottom }}-\dot{\epsilon}_{\text {top }}$ is the difference of growth rate between both sides, $\dot{\epsilon}_{\text {mean }}$ is the mean growth rate and $\tau_{g}=\dot{\epsilon}_{\text {mean }}^{-1}$ is the growth timescale (Silk, 1984; Moulia and Fournier, 2009; Bastien et al., 2013, Figure 1). The growth rate of plant cells is known to be controlled by auxin, the so-called growth hormone. Auxin stimulates cell elongation by loosening cell walls. To the best of our knowledge, the link between the local auxin concentration in walls and the local growth rate of cells has not been robustly determined and only the response of the whole tissue to an external addition of auxin has been investigated. It is however often assumed that growth is mainly controlled by the auxin concentration in the vicinity of the "skin" of the organ, as epidermal tissues are stiffer than inner tissues (Kutschera and Niklas, 2007; Dyson et al., 2014). For the sake of simplicity, we will here assume that the local growth rate is simply proportional to the local auxin concentration $c, \dot{\epsilon}=k c$ (Galston and Hand, 1949; Hopkins and Hüner, 2009), such that:

$$
R \frac{\mathrm{d} \mathcal{C}}{\mathrm{d} t}=\frac{1}{\tau_{g}} \times \frac{c_{\text {bottom }}-c_{\mathrm{top}}}{2 c_{\text {mean }}}
$$

where $c_{\text {bottom }}$ and $c_{\text {top }}$ are the auxin concentrations on both sides of the organ and $c_{\text {mean }}$ the mean auxin concentration. Under this assumption, the dimensionless gravitropic response deduced from the curvature dynamics, $\tilde{\Delta}(t) \equiv R \tau_{g} \frac{d \mathcal{C}}{d t}$, is equal to the relative auxin gradient across the organ:

$$
\tilde{\Delta}(t)=\frac{c_{\text {bottom }}-c_{\text {top }}}{2 c_{\text {mean }}} .
$$

The goal of the model is to predict how this auxin gradient establishes when the plant is tilted.

\subsection{Auxin Transport}

Auxin transport plays a key role in shaping plants development and, as such, has been the topic of extensive research over the past decades. Auxin transport is based on two distinct mechanisms (Goldsmith, 1977; Hopkins and Hüner, 2009; Runions et al., 2014). On the one hand, auxin in cell walls (mostly in a protonated form) enters the neighboring cell passively, or thanks to Aux/Lax influx carriers that are evenly distributed throughout the membrane. On the other hand, auxin inside cells (mostly in an anionic form) can only exit thanks to active auxin efflux carriers, such as PIN proteins (Krecek et al., 2009) or ABCB transporters (Zažímalová et al., 2010). While ABCB are evenly distributed throughout the membrane, PIN proteins are usually polarized and can be redistributed in response to external stimuli such as gravity (in particular PIN3, which is known to be implied in gravitropic response, see Friml et al., 2002; Rakusová et al., 2011). Hence, an asymmetric distribution of PIN carriers on each side of the cell can generate an active transport of auxin from one cell to the other, resulting in a stable auxin gradient.

To model this situation, we provide a simplified description of auxin transport in which the different forms of auxin (protonassociated or not) are not taken into account. The tissue across the shoot or stem (width $2 R$ ) is modeled as a one-dimensional array of $N$ cells of width $W$, separated by a cell wall of width $w$ (Figures 1B, 2). We denote $c_{n}$ the auxin concentration inside the $n$-th cell and $C_{n}$ the auxin concentration inside the $n$-th wall, which are both assumed uniform (the equilibrium time of auxin in each compartment is very fast, about $0.1 \mathrm{~s}$ in the cell wall and few s inside the cell taking typical values of auxin diffusion coefficients, see Kramer et al., 2007; Grieneisen et al., 2012). We also neglect auxin dilution due to cell growth and assume that auxin is neither degraded nor created, as the degradation time (of the order 1-3 h) and the replacement time (of the order 3-10 h) of auxin are longer than the timescales $(<1 \mathrm{~h})$ we are interested in Kramer and Ackelsberg (2015). The efflux current of auxin (number of auxin molecules per unit time and unit surface) from the $n$-th cell to the left wall (resp. right wall) is given by $P_{n}^{l} c_{n}$ (resp. $P_{n}^{r} c_{n}$ ), where $P_{n}^{l}$ (resp. $P_{n}^{r}$ ) is the permeability of the left (resp. right) membrane (unit $\mathrm{m} / \mathrm{s}$ ). Conversely, the influx current of auxin from the $n$-th wall to both adjacent cells is $P{ }^{\text {in }} C_{n}$, where the influx permeability $P^{\text {in }}$ is assumed uniform for all cells (see Figure 2). The time-evolution of the concentration is then:

$$
\begin{aligned}
& w \frac{\mathrm{d} C_{n}}{\mathrm{~d} t}=-2 P^{\mathrm{in}} C_{n}+P_{n}^{r} c_{n}+P_{n+1}^{l} c_{n+1}, \\
& W \frac{\mathrm{d} c_{n}}{\mathrm{~d} t}=-\left(P_{n}^{l}+P_{n}^{r}\right) c_{n}+P^{\mathrm{in}}\left(C_{n-1}+C_{n}\right) .
\end{aligned}
$$

In these expression, there is no vertical flux, as in this 1D model we assume invariance along the longitudinal (stem) axis. The cell wall size being much smaller than the cell width $(w \ll W)$, the auxin concentration in the cell wall can be assumed quasi-steady, 


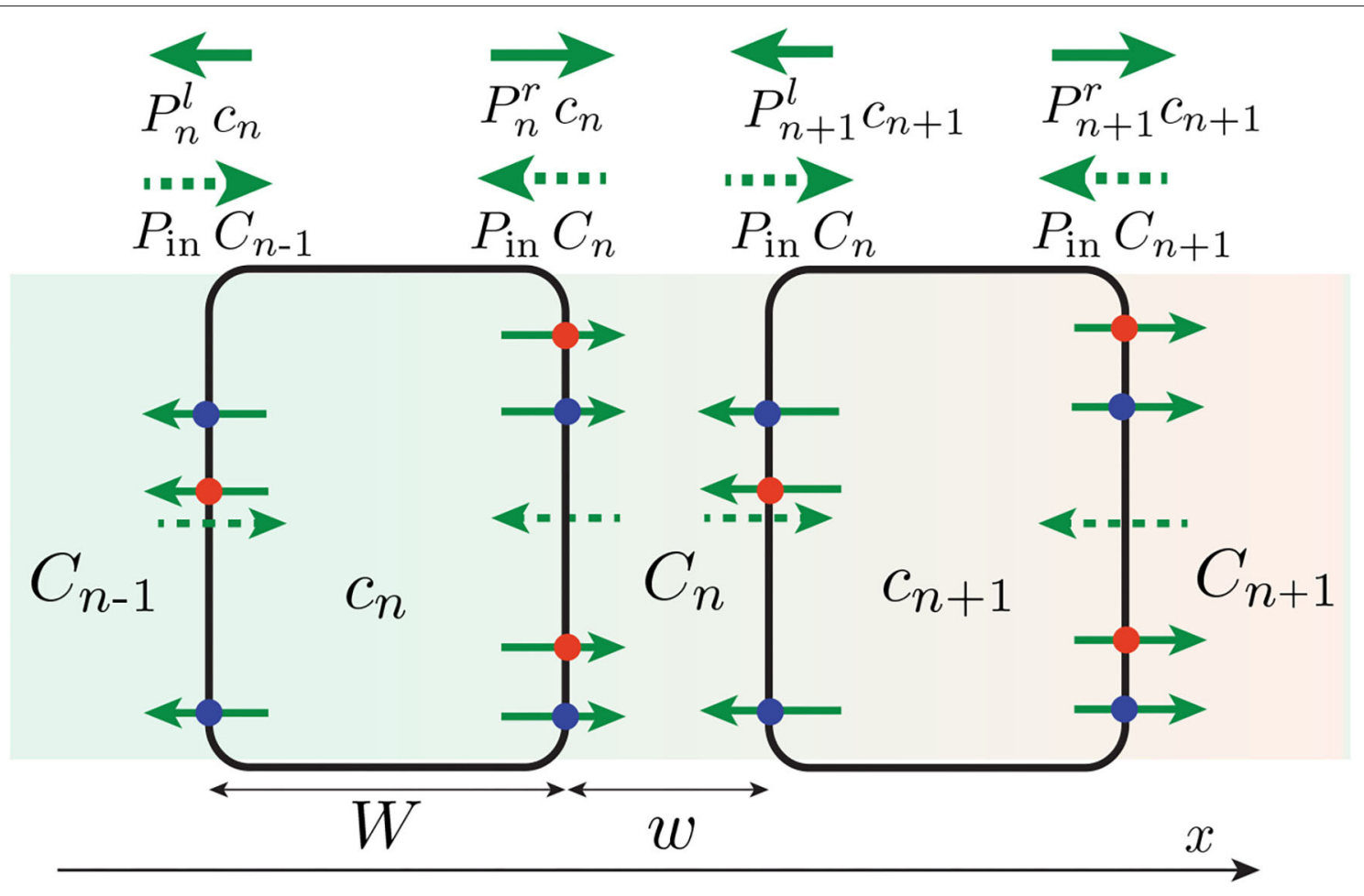

FIGURE 2 | One-dimensional, discrete model of auxin transport across the tissue (in reality $w \ll W$ ). Efflux of auxin (solid green arrow) occurs through efflux carriers (PIN: red circle, ABCB: blue circle), whose distribution (and thus permeabilities $P_{n}^{\prime, r}$ ) can be different on the right and left membrane of the cell. By contrast, influx of auxin (green dotted arrow) occurs with a symmetrical permeability $P^{\text {in }}$ on both side of the cell. An asymmetry of efflux permeabilities $P^{\prime} \neq P^{r}$ can generate a net flux of auxin across the tissue, yielding an auxin concentration gradient (background color gradient).

$2 P^{\text {in }} C_{n} \simeq P_{n}^{r} c_{n}+P_{n+1}^{l} c_{n+1}$, yielding:

$$
2 W \frac{\mathrm{d} c_{n}}{\mathrm{~d} t}=P_{n+1}^{l} c_{n+1}-P_{n}^{l} c_{n}+P_{n-1}^{r} c_{n-1}-P_{n}^{r} c_{n} .
$$

In the following, we assume that the distribution of auxin efflux carriers is the same in each cell, so that $P^{l}$ and $P^{r}$ are independent of $n$. This is the case of shoot coleoptiles where all cells in the growing region are similar and contain statoliths (Bérut et al., 2018), but not the case of stems like the inflorescence of Arabidopsis, where statoliths are only present on an external ring in the endodermal cells (the modification of the equation in this case of inhomogeneous tissue is given in Appendix A). We also assume that auxin gradients occur over a length scale much larger than the cell size. In the continuum limit $\left[c_{n}(t) \rightarrow c(x, t)\right.$, $c_{n+1}(t) \rightarrow c(x+W, t) \approx c(x, t)+W \frac{\partial c}{\partial x}+\frac{W^{2}}{2} \frac{\partial^{2} c}{\partial x^{2}}, c_{n-1}(t) \rightarrow$ $\left.c(x-W, t) \approx c(x, t)-W \frac{\partial c}{\partial x}+\frac{W^{2}}{2} \frac{\partial^{2} c}{\partial x^{2}}\right]$, Equation (5) for auxin transport then reduces to:

$$
\frac{\partial c}{\partial t}=\left(\frac{P^{l}+P^{r}}{2}\right) \frac{W}{2} \frac{\partial^{2} c}{\partial x^{2}}-\left(\frac{P^{r}-P^{l}}{2}\right) \frac{\partial c}{\partial x}
$$

One recognizes an advection-diffusion equation:

$$
\frac{\partial c}{\partial t}=\mathcal{D} \frac{\partial^{2} c}{\partial x^{2}}-v \frac{\partial c}{\partial x}
$$

with a coefficient of diffusion given by $\mathcal{D}=W P / 2$, where $P=$ $\left(P^{l}+P^{r}\right) / 2$, and an advection speed given by $v=\delta P / 2$, where $\delta P=P^{r}-P^{l}$.

The advective part of Equation (7), which is responsible for auxin transport from one side to the other and thus to the differentiated growth and the curvature of the organ, is entirely controlled by the asymmetry of efflux permeabilities $\delta P$. Since ABCB carriers are evenly distributed, this asymmetry solely comes from the asymmetry of PINs distribution between the right and left side of the cells. The advection speed is thus given by:

$$
v=\frac{\delta P}{2}=\frac{P^{r}-P^{l}}{2}=\frac{1}{2 S}\left(\alpha \int_{S}[P I N]_{\mid \text {right }}-\alpha \int_{S}[P I N]_{\mid \text {left }}\right),
$$

where $\alpha$ is the conductance of a single PIN carrier (unit $\left.\mathrm{m}^{3} / \mathrm{s}\right), S$ the lateral surface area of the cells and $[P I N]$ the surface concentration of PINs attached to the membrane. The coefficient of diffusion $\mathcal{D}$, on the other hand, is given by:

$$
\mathcal{D}=\frac{W P}{2}=\frac{W}{4 S}\left(\alpha \int_{S}[P I N]_{\mid \text {right }}+\alpha \int_{S}[P I N]_{\mid \text {left }}+2 \beta \int_{S}[A B C B]\right),
$$




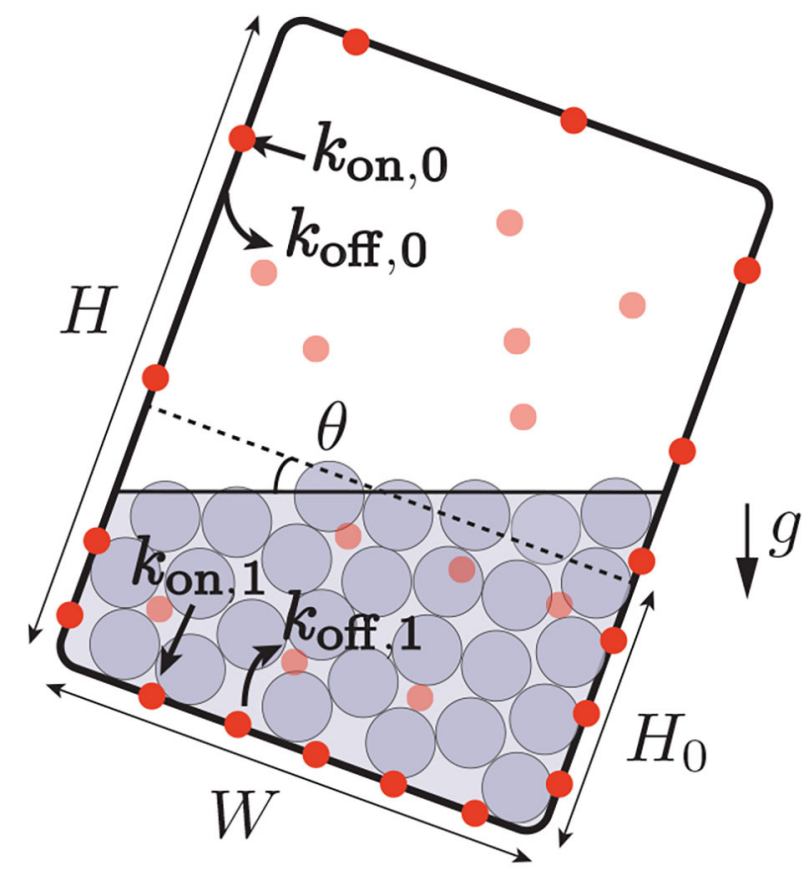

FIGURE 3 | Interaction between PIN trafficking and statoliths position. The rate of reallocation $k_{\text {on }}$ and deallocation $k_{\text {off }}$ of PINs (bold red circles: PINs attached to the cell membrane, light red circles: PINs in bulk) depends on the presence of statoliths (gray). When the cell is tilted, the asymmetric distribution of the position of the statoliths induces a bias in the distribution of the PINs attached to the membrane.

where $\beta$ is the conductance of a single $\mathrm{ABCB}$ carrier (unit $\mathrm{m}^{3} / \mathrm{s}$ ). The coefficient of diffusion depends on the total number of efflux carriers (ABCB and $\mathrm{PIN}$ ) on the cell membrane and is thus weakly affected by the PIN asymmetry. In the following, we assume that $\mathcal{D}$ is constant, which simplifies the results without affecting much the conclusions.

\subsection{Coupling PIN Dynamics to Statoliths Position: Biased Efflux at Cell Scale}

The previous section relates auxin transport to the asymmetry of PINs distribution at the cellular level. We now model how this asymmetry emerges when the plant is tilted under gravity. Recently, it has been demonstrated that the relevant gravitropic stimulus for graviperception is the statoliths position within the statocytes (position-sensor hypothesis), and not their weight as previously believed (Chauvet et al., 2016; Pouliquen et al., 2017). Statoliths have also been identified as key actors in the relocalization of PIN-proteins in response to change of gravity direction in both roots and shoots (Friml et al., 2002; Rakusová et al., 2011; Grones et al., 2018; Nakamura et al., 2019b). Yet, how statoliths position is detected and read to modify PIN polarity remains largely unknown. PINs trafficking involves synthesis in the endoplasmic reticulum, degradation in the vacuole and recycling (Kleine-Vehn and Friml, 2008). Recycling is achieved by endocytosis, i.e., the deallocation of PIN proteins formerly attached to the cell membrane toward the cytoplasm inside a vesicle, or by exocytosis, i.e., the reallocation of the vesicle-carried PINs from the cytoplasm back to the cell membrane.

Following the position-sensor hypothesis, we assume that the presence of statoliths, either through direct steric constraints or through indirect molecular signaling, modify the trafficking of PIN proteins, so that PINs polarize preferentially on places where statoliths are in contact with it. This mechanism is formalized as follows. The endocytosis rate of PINs, $\mathrm{d}[P I N]_{i} /\left.\mathrm{d} t\right|_{\text {endo }}=$ $-k_{\mathrm{off}, i}[\mathrm{PIN}]_{i}$ where $[P I N]_{i}$ is the surface concentration of PIN attached to the membrane, is assumed to depend on the presence of statoliths, with $i=0$ if no statoliths are present and $i=$ 1 if they are (see Figure 3). Similarly, the rate of exocytosis is written as $\mathrm{d}[P I N]_{i} /\left.\mathrm{d} t\right|_{\text {exo }}=+k_{\text {on }, i}[P I N]_{\mathrm{vol}}$, where $[P I N]_{\mathrm{vol}}$ is the volumic concentration of PINs molecules inside the cell of volume WS. Two cases will be distinguished in the model, depending on whether PINs can attach to any side of the cell ("apical/basal/lateral binding") or only on lateral sides ("lateral binding"; see Figure 4A). Assuming that the total number $N_{\text {tot }}$ of PINs is conserved during gravistimulation (Kleine-Vehn et al., 2010; Rakusová et al., 2011) leads to the following set of equations for the PIN concentration attached to the membrane, $[P I N]_{i=0,1}$ :

$$
\begin{aligned}
& \frac{\mathrm{d}[P I N]_{0}}{\mathrm{~d} t}=-k_{\mathrm{off}, 0}[P I N]_{0} \\
& +k_{\mathrm{on}, 0}\left(\frac{N_{\mathrm{tot}}-\left(S_{0}[P I N]_{0}+S_{1}[P I N]_{1}\right)}{W S}\right), \\
& \frac{\mathrm{d}[P I N]_{1}}{\mathrm{~d} t}=-k_{\mathrm{off}, 1}[P I N]_{1} \\
& +k_{\mathrm{on}, 1}\left(\frac{N_{\mathrm{tot}}-\left(S_{0}[P I N]_{0}+S_{1}[P I N]_{1}\right)}{W S}\right),
\end{aligned}
$$

where $S_{1}$ (resp. $S_{0}$ ) denotes the total surface area in contact (resp. not in contact) with statoliths in case of apical/basal/lateral binding, or only the lateral surfaces in contact (resp. not in contact) with statoliths in the lateral binding case.

The form of Equation (10) shows that two regimes can be distinguished. The first regime, called "infinite-pool" regime in the following, corresponds to the case where $N_{\text {tot }}$ is large compared to $S_{0}[P I N]_{0}+S_{1}[P I N]_{1}$, such that PINs concentration in the bulk can be assumed constant and not affected by PINs attachment at the cell membrane. The second regime, called "limiting-pool" regime, corresponds to the opposite situation where most of the PINs are attached to the cell membrane, such that $S_{0}[P I N]_{0}+S_{1}[P I N]_{1} \simeq N_{\text {tot }}$. We will see in the next section that these regimes are controlled by the pool-number $\mathcal{P}$ defined by:

$$
\mathcal{P}=\frac{W k_{\mathrm{off}, 1}}{k_{\mathrm{on}, 1}},
$$

where $\mathcal{P} \gg 1$ corresponds to the infinite-pool regime and $\mathcal{P} \ll 1$ corresponds to the limiting-pool regime (see Figure 4B). Note that in writing Equation (10), we have neglected the diffusion of PINs inside the membrane. This is justified since, over the time scales we are interesting in (running from minutes to $1 \mathrm{~h}$ ), PINs 
A

A/B/L binding

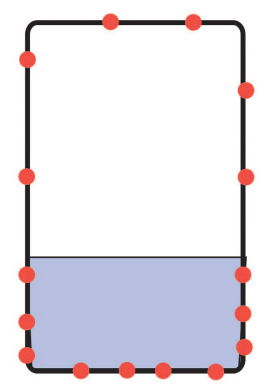

L-binding

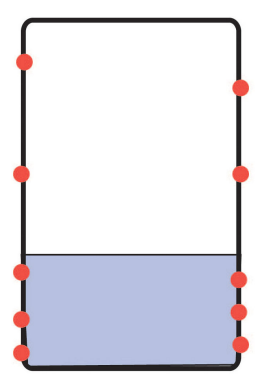

B

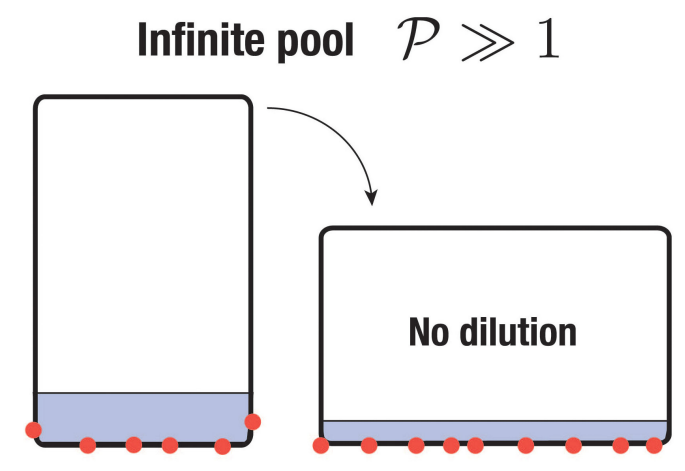

Limiting pool $\mathcal{P} \ll 1$
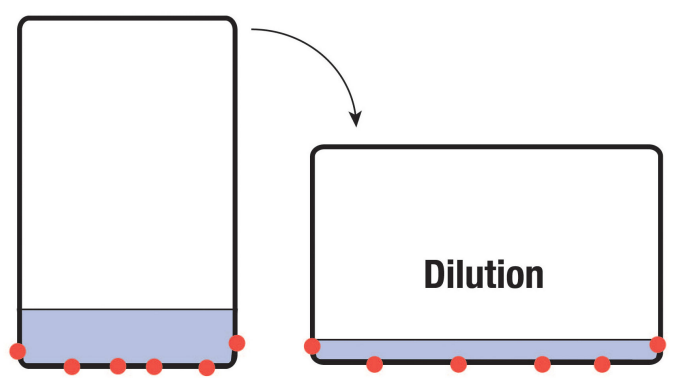

C

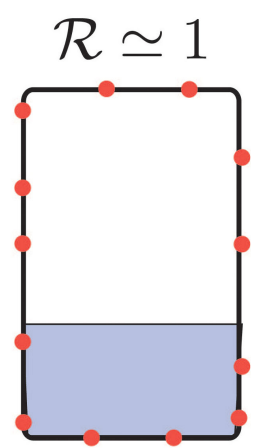

$\mathcal{R} \gg 1$

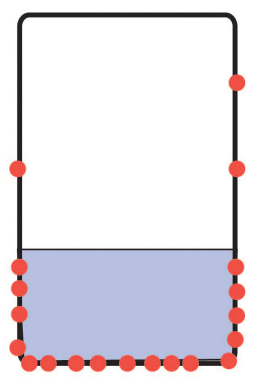

FIGURE 4 | Sketch of different scenario of PIN-binding. PINs are represented in red and the region with statoliths in gray. (A) Apical/basal/lateral (A/B/L) binding vs. lateral (L) binding. (B) Infinite pool versus limiting pool. In the first case, the surface density of PINs is conserved whereas in the second one, the total number of PINs attached to the cell membrane is conserved. (C) Low sensitivity of PIN to statoliths $(\mathcal{R} \simeq 1)$ or high sensitivity $(\mathcal{R} \gg 1)$.

diffuse only over a distance of about few micrometers, which is much smaller than the cell size (taking $0.1 \mu \mathrm{m}^{2}$ min for the diffusion coefficient of a PIN, see Kleine-Vehn et al., 2011).

Equations $(1,7,10)$ represent a complete model describing the time-evolution of the gravitropic response once the statoliths position is known, from the PIN localization to auxin transport and bending of the plant. In the following, we study the predictions of the model for a steady and transient stimulus. Tables 1, 2 summarize all the physical quantities and dimensionless parameters defined from the model, while Table 3 gathers the assumptions made in the model.

\section{RESULTS}

\subsection{Steady Gravitropic Response: Revisiting the Sine-Law}

We first study the gravitropic response predicted by our model in the case of a steady inclination of the plant $\theta$, for long timescales when the system reaches a steady state. This situation corresponds to the usual protocol for measuring the sensitivity of plant to gravity under steady condition, when the plant is suddenly inclined to a fixed angle $\theta$ and its curvature (or tip angle) measured over time. After a transient, the rate of change of curvature is found to be constant (Chauvet et al., 2016), which enables to measure the steady gravitropic response $\tilde{\Delta}^{\text {steady }}(\theta)=$ $R \tau_{g} \frac{\mathrm{d} \mathcal{C}}{\mathrm{d} t}$ (see section 2) for each imposed angle $\theta$. For many plants, this relationship between the gravitropic response and the inclination angle has sine-like shape (the response is null for $\theta=0^{\circ}$ or $\theta=180^{\circ}$ and maximal for $\theta=90^{\circ}$ ) and is called the "sine-law" in the literature (Sachs, 1887; Larsen, 1969; Iino et al., 1996; Galland, 2002; Dumais, 2013). Below, we determine the steady gravitropic response $\tilde{\Delta}^{\text {steady }}(\theta)$ predicted by the model and compare with measurements of the sine-law obtained previously for wheat coleoptiles over a wide range of angles (Chauvet et al., 2016).

In the steady regime, the auxin transport equation (Equation 7) reduces to: $\mathrm{d} J / \mathrm{d} x=0$, where $J=\mathcal{D}(\mathrm{d} c / \mathrm{d} x)-v c$ is the auxin flux. For impermeable boundaries at $x=0$ and $x=2 R$, the flux is null $(J=0)$ and the auxin concentration profile is then given by:

$$
c(x)=c_{\text {mean }} \frac{\mathrm{Pe} \times \exp \left(\mathrm{Pe} \frac{x}{2 R}\right)}{\exp (\mathrm{Pe})-1}
$$

where $c_{\text {mean }}=(1 / 2 R) \int_{0}^{2 R} c(x) \mathrm{d} x$ is the mean concentration of auxin and $\mathrm{Pe}$ is the Peclet number defined by:

$$
\mathrm{Pe} \equiv \frac{2 R v}{\mathcal{D}}=\frac{\alpha N W}{2 \mathcal{D} S}\left(\int_{S}[P I N]_{\mid \text {right }}-\int_{S}[P I N]_{\mid \text {left }}\right),
$$

using the expression (8) of $v$. The shape of the auxin concentration profile is determined by the value of the Peclet number. For $\mathrm{Pe} \ll 1$, the profile is linear and the auxin level in the 
TABLE 1 | List of dimensional quantities in the model with their definition and unit.

\begin{tabular}{|c|c|c|}
\hline $\begin{array}{l}\text { Physical } \\
\text { quantities }\end{array}$ & Definition & Unit \\
\hline$R$ & Radius of the organ & (m) \\
\hline $\mathcal{C}$ & Bending curvature of the organ & $\left(m^{-1}\right)$ \\
\hline W & Cell width & (m) \\
\hline$w$ & Cell wall width & (m) \\
\hline$H$ & Cell height & (m) \\
\hline$H_{\text {stato }}$ & Statolith pile height before inclination & (m) \\
\hline$s$ & Cell lateral surface area & $\left(m^{2}\right)$ \\
\hline$S_{0,1}$ & $\begin{array}{l}\text { Total surface area of the cell not (0) or } \\
\text { in contact (1) with statoliths }\end{array}$ & $\left(m^{2}\right)$ \\
\hline$S_{0}^{\prime, r}$ & $\begin{array}{l}\text { Surface area of the left }(\eta) \text { or right }(r) \\
\text { side of the cell not in contact with } \\
\text { statoliths }\end{array}$ & $\left(m^{2}\right)$ \\
\hline$S_{1}^{\prime, r}$ & $\begin{array}{l}\text { Surface area of the left }(\eta) \text { or right }(r) \\
\text { side of the cell in contact with } \\
\text { statoliths }\end{array}$ & $\left(m^{2}\right)$ \\
\hline$\dot{\epsilon}$ & Growth rate & $\left(s^{-1}\right)$ \\
\hline$\tau_{g}$ & $\tau_{g}=\dot{\epsilon}_{\text {mean }}^{-1}$ growth timescale & (s) \\
\hline$c$ & Auxin concentration inside the cell & $\left(\mathrm{mol} \mathrm{m}^{-3}\right)$ \\
\hline C & Auxin concentration in the cell wall & $\left(\mathrm{mol} \mathrm{m}^{-3}\right)$ \\
\hline$P^{l, r}$ & $\begin{array}{l}\text { Auxin efflux permeability of the left (I) } \\
\text { or right }(r) \text { side of the cell }\end{array}$ & $\left(\mathrm{m} \mathrm{s}^{-1}\right)$ \\
\hline$P^{\text {in }}$ & Auxin influx permeability & $\left(\mathrm{m} \mathrm{s}^{-1}\right)$ \\
\hline$\alpha$ & Conductance of a single PIN carrier & $\left(m^{3} \mathrm{~s}^{-1}\right)$ \\
\hline$\beta$ & Conductance of a single $\mathrm{ABCB}$ carrier & $\left(m^{3} s^{-1}\right)$ \\
\hline$P$ & $\begin{array}{l}P=\frac{P^{\prime}+P^{r}}{2}= \\
\frac{1}{2 S}\left(\alpha \int_{S}[P I N]_{\mid \text {right }}+\alpha \int_{S}[P I N]_{\| \text {eft }}+2 \beta \int_{S}[A B C B]\right)\end{array}$ & $\left(\mathrm{m} \mathrm{s}^{-1}\right)$ \\
\hline$\delta P$ & $\begin{array}{l}\delta P=P^{r}-P^{\prime}= \\
\frac{1}{S}\left(\alpha \int_{S}[P I N]_{\mid \text {right }}-\alpha \int_{S}[P I N]_{\mid \text {left }}\right)\end{array}$ & $\left(\mathrm{m} \mathrm{s}^{-1}\right)$ \\
\hline $\mathcal{D}$ & Auxin coefficient of diffusion $\mathcal{D}=\frac{W P}{2}$ & $\left(m^{2} s^{-1}\right)$ \\
\hline$v$ & Auxin advection speed $v=\frac{\delta P}{2}$ & $\left(\mathrm{~m} \mathrm{~s}^{-1}\right)$ \\
\hline$k_{\mathrm{off}, 0,1}$ & $\begin{array}{l}\text { Endocytosis rate when statoliths are } \\
\text { (1) or are not }(0) \text { in contact with the } \\
\text { membrane }\end{array}$ & $\left(s^{-1}\right)$ \\
\hline$k_{\mathrm{on}, 0,1}$ & $\begin{array}{l}\text { Exocytosis speed when statoliths are } \\
\text { (1) or are not }(0) \text { in contact with the } \\
\text { membrane }\end{array}$ & $\left(\mathrm{m} \mathrm{s}^{-1}\right)$ \\
\hline$\tau_{\text {aux }}$ & $\begin{array}{l}\text { Timescale for auxin transport across } \\
\text { the tissue } \tau_{\text {aux }}=\frac{1}{\pi^{2}+\left(\mathrm{Pe}^{2} / 4\right)} \times \frac{(2 R)^{2}}{\mathcal{D}}\end{array}$ & (s) \\
\hline$\tau_{\mathrm{PIN}}$ & $\begin{array}{l}\text { Timescale for PIN turnover } \\
\tau_{\mathrm{PIN}}=\frac{k_{\mathrm{on}, 0} S_{0}+k_{\mathrm{on}, 1} S_{1}}{k_{\mathrm{off}, 0} k_{\mathrm{on}, 1} S_{1}+k_{\mathrm{off}} k_{\mathrm{on}, 0} S_{0}}\end{array}$ & (s) \\
\hline
\end{tabular}

middle of the stem is unchanged, whereas for $\mathrm{Pe} \gg 1$ the profile is strongly asymmetric with most auxin concentrated on the right, corresponding to the lower side of the shoot (Figure 5). From this steady profile of auxin, the gravitropic response can be computed as $\tilde{\Delta}^{\text {steady }}=\frac{c(x=2 R)-c(x=0)}{2 c_{\text {mean }}}$ (Equation 2$)$, which gives:

$$
\tilde{\Delta}^{\text {steady }}=\frac{\mathrm{Pe}}{2} \text {. }
$$

In the steady state, the gravitropic response of the plant is thus given by the value of the Peclet number. Previous measurements in various plant species representative of land angiosperm
TABLE 2 | List of the dimensionless parameters used in the model with their definition and meaning.

\begin{tabular}{|c|c|c|}
\hline $\begin{array}{l}\text { Dimensionless } \\
\text { parameters }\end{array}$ & Definition & Meaning \\
\hline$\theta$ & & Inclination of the plant \\
\hline$N$ & & $\begin{array}{l}\text { Number of cells across the } \\
\text { tissue }\end{array}$ \\
\hline$N_{\text {tot }}$ & & $\begin{array}{l}\text { Total number of PIN carriers } \\
\text { per cell }\end{array}$ \\
\hline$\tilde{\Delta}$ & $\tilde{\Delta}=\frac{C_{\text {bottom }}-C_{\text {top }}}{2 C_{\text {mean }}}$ & Gravitropic response \\
\hline $\mathrm{Pe}$ & $\mathrm{Pe}=\frac{2 R v}{\mathcal{D}}$ & $\begin{array}{l}\text { Peclet number comparing } \\
\text { auxin advection to diffusion }\end{array}$ \\
\hline $\mathcal{A}$ & $\mathcal{A}=\frac{\alpha N_{\text {tot }} W N}{2 S \mathcal{D}}$ & $\begin{array}{l}\text { Ratio of PIN to total efflux } \\
\text { carrier conductance } \\
\text { multiplied by } N\end{array}$ \\
\hline $\mathcal{R}$ & $\mathcal{R}=\frac{\left[P \mathbb{N}_{1}^{\text {steady }}\right.}{\left[P \mathbb{N}_{0}^{\text {steady }}\right.}=\frac{k_{\text {on }, 1} k_{\text {offi, }}}{k_{\text {on, } 0} k_{\text {offi, }}}$ & $\begin{array}{l}\text { Statoliths/PIN coupling } \\
\text { parameter }\end{array}$ \\
\hline $\mathcal{P}$ & $\mathcal{P}=\frac{W_{k_{\mathrm{off}, 1}}}{k_{\mathrm{on}, 1}}$ & $\begin{array}{l}\text { Ratio of endocytosis to } \\
\text { exocytosis (pool number) }\end{array}$ \\
\hline
\end{tabular}

showed that $\tilde{\Delta}^{\text {steady }}$ is typically of the order 1 (Chauvet et al., 2016 , for e.g., in wheat coleoptile, the maximal value of $\tilde{\Delta}^{\text {steady }}$ obtained for a 90 degrees inclination is about 0.7 ), meaning that the Peclet number is typically of the order 1 . Therefore, the auxin profile across the shoot is expected to be close to linear (Figure 5A, and not a pronounced exponential, Figure 5B). A consequence is that growth, which was assumed proportional to the auxin concentration, varies also linearly from one side of the shoot to the other during the gravitropic response.

The next step to determine $\tilde{\Delta}^{\text {steady }}$ is to compute the Peclet number given by Equation (13), i.e., the relative distribution of PIN between the left and right side of the cell. In the steady regime, the concentration of PIN attached to a membrane not covered by statoliths $\left([P I N]_{0}^{\text {steady }}\right)$, or covered by statoliths $\left([P I N]_{1}^{\text {steady }}\right)$, is given by (see Equation 10$)$ :

$$
\begin{aligned}
& {[P I N]_{0}^{\text {steady }}=\frac{N_{\text {tot }}}{S}\left(\frac{k_{\mathrm{off}, 0} W}{k_{\mathrm{on}, 0}}+\frac{1}{S}\left[S_{0}+\frac{k_{\mathrm{on}, 1} k_{\mathrm{off}, 0}}{k_{\mathrm{on}, 0} k_{\mathrm{off}, 1}} S_{1}\right]\right)^{-1},} \\
& {[P I N]_{1}^{\text {steady }}=\frac{N_{\mathrm{tot}}}{S}\left(\frac{k_{\mathrm{off}, 1} W}{k_{\mathrm{on}, 1}}+\frac{1}{S}\left[S_{1}+\frac{k_{\mathrm{on}, 0} k_{\mathrm{off}, 1}}{k_{\mathrm{on}, 1} k_{\mathrm{off}, 0}} S_{0}\right]\right)^{-1} .}
\end{aligned}
$$

Noting $S_{i}^{l}$ (resp. $S_{i}^{r}$ ) the surface area of the left (resp. right) side of the cell not covered $(i=0)$ or covered $(i=1)$ by statoliths, we have $\int_{S}[P I N]_{\mid \text {right }}-\int_{S}[P I N]_{\mid \text {left }}=[P I N]_{0}^{\text {steady }}\left(S_{0}^{r}-S_{0}^{l}\right)+$ $[P I N]_{1}^{\text {steady }}\left(S_{1}^{r}-S_{1}^{l}\right)$. Finally, since $S_{0}^{l}+S_{1}^{l}=S_{0}^{r}+S_{1}^{r}=S$, where $S$ is the lateral surface area of the cells and using Equation (13), we have:

$$
\tilde{\Delta}^{\text {steady }}=\frac{\alpha W N}{4 \mathcal{D S}}(\mathcal{R}-1)\left(S_{1}^{r}-S_{1}^{l}\right)[P I N]_{0}^{\text {steady }}
$$


TABLE 3 | Assumptions used in the model and possible improvements/extensions.

\section{Geometry and growth}

- 1D tissue

- Homogeneous properties (cell shape, statoliths content, auxin carriers properties, and growth capabilities)

- Growth rate proportional to local auxin concentration (Galston and Hand, 1949; Hopkins and Hüner, 2009)

\section{Auxin transport}

- 1D transport (transverse gradient only, no longitudinal flux and gradient along the organ)

- No production or degradation of auxin

(Kramer and Ackelsberg, 2015)

- Auxin concentration uniform in each cell and cell wall

(Kramer et al., 2007; Grieneisen et al., 2012)

- Continuum approximation: auxin gradients occurs on a length scale larger than the cell size

- Coefficient of diffusion of auxin $\mathcal{D}$ not affected by PINs polarization

\section{PIN trafficking}

- Conservation of PINs upon gravistimulation (Kleine-Vehn et al., 2010; Rakusová et al., 2011)

- Statoliths modify PINs exocytosis ( $\left.k_{\circ}\right)$ and endocytosis

$\left(k_{\text {off }}\right)$ rate to favor cell membrane attachment $(\mathcal{R}>1)$

- PIN conductance $\alpha$ not affected by statoliths

- PINs diffusion inside the cell membrane neglected (Kleine-Vehn et al., 2011)

- PINs bulk concentration uniform inside the cells

\section{Statoliths dynamics}

- Liquid behavior of the statoliths pile (horizontal free surface in steady state) (Bérut et al., 2018)

- Neglect the duration of statoliths repositioning compared

to the gravitropic response time (Chauvet et al., 2019)

- Saltating (fluctuating) motion of statoliths neglected
- 2D and 3D tissue (Dyson et al., 2014)

- Introducing a spatial variability (distribution) or inhomogeneity in cell and tissue properties

- More complex growth model coupled with the cell wall rheology (e.g., Dyson et al., 2014)

- 2D and 3D auxin transport model (e.g., Fendrych et al., 2018; Retzer et al., 2019)

- Adding auxin sink and source

- Use of discrete (cellular) models

- Use of the exact expression (9) in the algebra
- See section 4.3

- Taking into account intracellular heterogeneities (vacuole, cytoskeleton, etc)

References in the left column are those that support the assumptions; references in the right column are related to the proposed extensions.

with

$$
[P I N]_{0}^{\text {steady }}=\frac{N_{\text {tot }}}{S} \frac{1}{\mathcal{R}}\left(\mathcal{P}+\frac{S_{1}}{S}\left(1+\frac{S_{0}}{S_{1} \mathcal{R}}\right)\right)^{-1},
$$

and:

$$
\mathcal{R}=\frac{k_{\mathrm{on}, 1} k_{\mathrm{off}, 0}}{k_{\mathrm{on}, 0} k_{\mathrm{off}, 1}}=\frac{[P I N]_{1}^{\text {steady }}}{[P I N]_{0}^{\text {steady }}}, \quad \mathcal{P}=\frac{W k_{\mathrm{off}, 1}}{k_{\mathrm{on}, 1}} .
$$

The expressions (16-18) show that the steady gravitropic response is proportional to $(\mathcal{R}-1)\left(S_{1}^{r}-S_{1}^{l}\right)$. For $\theta>0$ as in Figure 1, the difference $\left(S_{1}^{r}-S_{1}^{l}\right)$ is positive since statoliths sediment toward the right side of the cell. Therefore, to obtain a "normal" gravitropic response $\left(\tilde{\Delta}^{\text {steady }}>0\right.$, i.e., a larger auxin concentration at the bottom side of the shoot), the ratio $\mathcal{R}$ must be larger than 1 . The parameter $\mathcal{R}$, called "statolith-PIN coupling" parameter in the following, characterizes the sensitivity of PIN to statoliths. In steady state, $\mathcal{R}$ is equal to the ratio between the concentration of PINs in a zone with statolith and in a zone without statolith: the larger $\mathcal{R}$, the more PINs in the region with statoliths compared to region without statolith (see Figure 4C). The other important parameter controlling the gravitropic response is the parameter $\mathcal{P}$. Combining Equations (17) and (18) shows that, in steady state: $N_{\text {tot }} /\left(S_{0}[P I N]_{0}+\right.$ $\left.S_{1}[P I N]_{1}\right)=1+\mathcal{P}\left[\left(S_{1} / S_{0}\right)+\left(S_{0} / S \mathcal{R}\right)\right]^{-1}$. Therefore, $\mathcal{P} \gg$ 1 corresponds to the infinite-pool regime $N_{\text {tot }} \gg S_{0}[P I N]_{0}+$ $S_{1}[P I N]_{1}$, whereas $\mathcal{P} \ll 1$ corresponds to the limiting-pool regime $N_{\text {tot }} \simeq S_{0}[P I N]_{0}+S_{1}[P I N]_{1}$, justifying the denomination of poolnumber for $\mathcal{P}$. Expression (18) shows that $\mathcal{P}$ is given by the ratio 

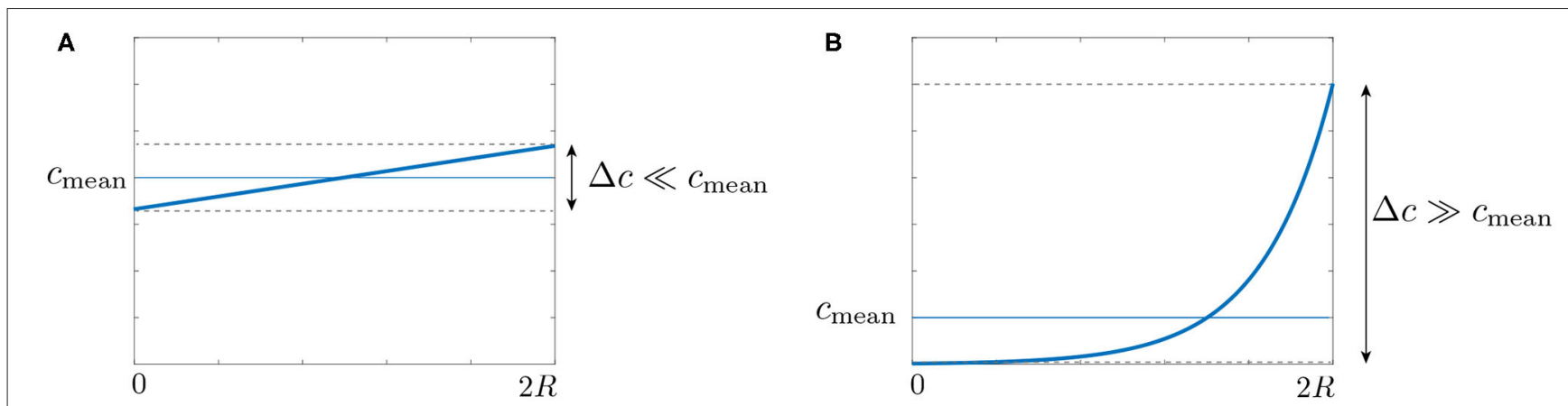

FIGURE 5 | Stationary auxin profile for (A) small and (B) large Peclet number.

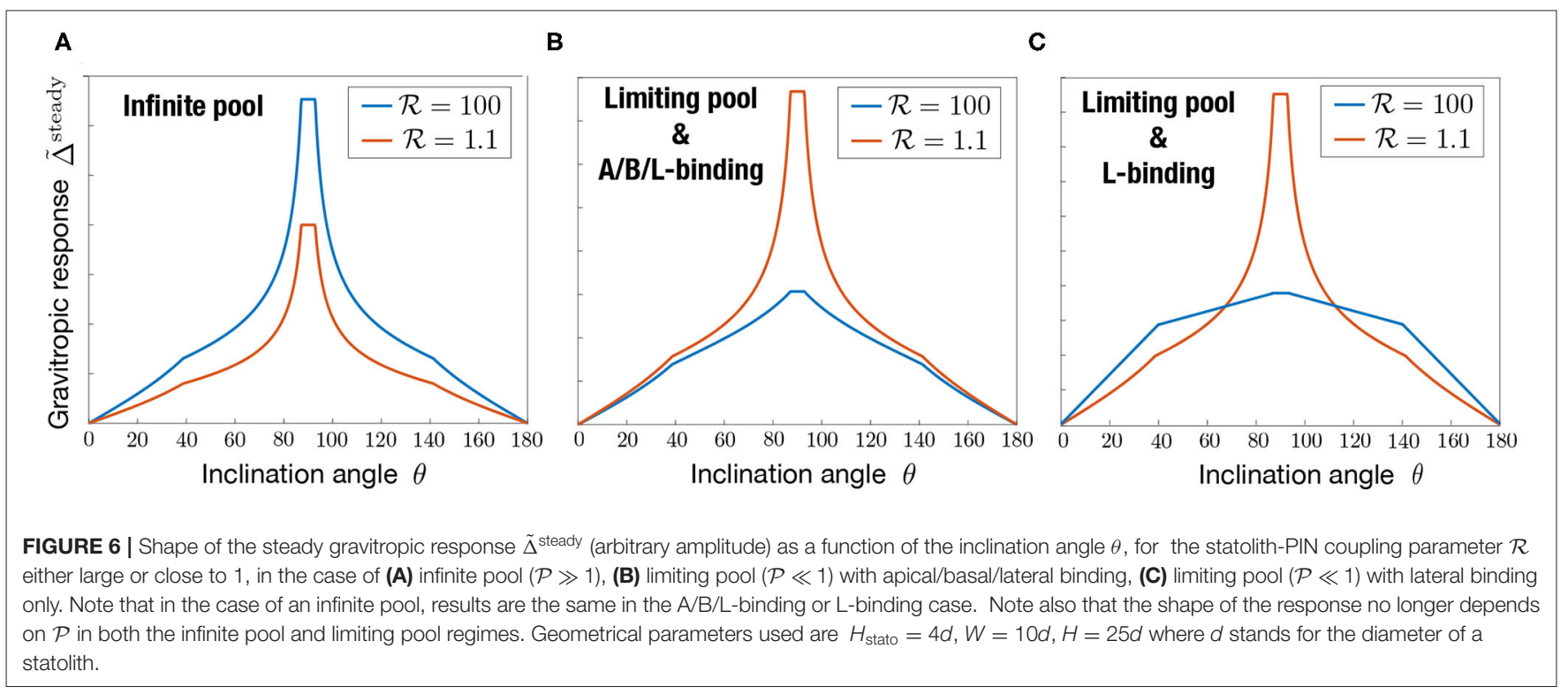

between the width of the cell $W$ and the length scale $k_{\mathrm{on}, 1} / k_{\mathrm{off}, 1}$, which can be interpreted as the distance of consumption of PINs in the bulk by exocytosis during the characteristic endocytosis time $k_{\text {off, } 1}^{-1}$. If this distance is small compared to the width of the cell, there is always enough PINs in the bulk to renew PINs in surface (infinite pool limit). In the opposite limit, PINs in the bulk are entirely consumed and all PINs are located on the cell membrane (limiting pool regime).

Once $\mathcal{R}$ and $\mathcal{P}$ are set, the final step is to compute how the different surfaces covered (and not covered) by the statoliths vary as function of the inclination angle $\theta$. To this end, one has to know the final position of statoliths when a cell is tilted. Recently, we addressed this question and showed that statoliths at the bottom of statocytes behave like an effective liquid on long timescale, due to the agitation of statoliths by the cell activity (Bérut et al., 2018). Therefore, the final free surface of the statoliths pile is horizontal, as sketched in Figure 3. This key feature of the flowing behavior of statoliths allows us to reduce the computation of the surfaces touched or not by the statoliths $\left(S_{0,1}^{r, l}, S_{0}\right.$, and $S_{1}$ in Equations 16,17$)$ to a purely geometrical problem, which depends on three parameters: the angle of inclination $\theta$, the aspect ratio of the cell $H / W$ and the initial aspect ratio of the statolith pile $H_{\text {stato }} / W$ (see Figure 3). The corresponding relationships are given in Appendix B.

Figure 6 presents the typical steady gravitropic response $\tilde{\Delta}^{\text {steady }}(\theta)$ predicted by the model (Equations $16,17,18$ ) as a function of $\theta$, in the case of an infinite pool $(\mathcal{P} \gg 1)$ or a limiting pool $(\mathcal{P} \ll 1)$, and for two extreme values of the statolithsPIN coupling parameter: $\mathcal{R} \simeq 1$ (red curve, low influence of statoliths on PIN binding) and $\mathcal{R} \gg 1$ (blue curve, strong influence of statoliths on PIN binding). The geometry used for the cell aspect ratio and the statoliths pile ratio is taken from experimental observations of wheat coleoptile statocytes, with a typical aspect ratio $H / W=2.5$ and $H_{\text {stato }} / W=1 / 2.5$. In the infinite pool regime (Figure 6A), the gravitropic response presents a convex shape with a strong peak close to $90^{\circ}$ whatever the value of $\mathcal{R}$, in disagreement with the usual sine-law shape. The amplitude of this peak in this regime also strongly depends on the cell geometry: the more elongated the cell, the higher the peak because in this case more lateral surface is available 
for PIN attachment without any PIN dilution (see Figure 4B). By contrast, in the limiting-pool regime (Figures $\mathbf{6 B}, \mathbf{C}$ ), the shape strongly depends on $\mathcal{R}$, the peak at $90^{\circ}$ being much more pronounced for $\mathcal{R} \simeq 1$ than for $\mathcal{R} \gg 1$. Interestingly, the response in this case also depends on whether PIN can attach on every sides of the cell (apical/basal/lateral binding) or only on the lateral sides (lateral binding case), as attachment to the "useless" apical and basal sides contribute to deplete PIN from the available pool. Overall, we see that only one case is compatible with the concave "sine-law" shape observed experimentally: a limiting pool of PIN with lateral binding and $\mathcal{R} \gg 1$ (blue curve in Figure 6C).

In the following, we thus assume that PIN recycling occurs in the limited-pool regime $(\mathcal{P} \ll 1)$, with lateral binding only. In this limit, and using expression of the surfaces given in Appendix B, the steady gravitropic response given by Equations $(16,17,18)$ can be written as:

\subsection{Transient Gravitropic Response: Dose-Response Law}

The previous results deal with the steady gravitropic response obtained when the gravity stimulus (the angle of inclination $\theta$ of the plant) is permanent. We now turn to the study of the transient gravitropic response, i.e., when the system has not yet reached the steady state. This situation typically corresponds to "dose-response" like experiments, in which the gravity stimulus is applied during a transient time $\Delta T$ only. Using such protocol on wheat coleoptiles, Chauvet et al. (2019) revealed the existence of an intrinsic "memory" time $\tau_{\text {memory }}$ in the gravitropic response. For $\Delta T \gg \tau_{\text {memory }}$, the response was constant and equal to the steady response $\tilde{\Delta}^{\text {steady }}$. However, for $\Delta T \lesssim \tau_{\text {memory }}$, the response was smaller and became proportional to $\Delta T$ (Figure 8). The memory time $\tau_{\text {memory }} \sim 15$ min identified in these experiments was longer than the sediment time of statoliths

$$
\tilde{\Delta}^{\text {steady }}(\theta)=\frac{1}{2} \mathcal{A}(\mathcal{R}-1)\left\{\begin{array}{l}
\frac{W \tan \theta}{2 H+2(\mathcal{R}-1) H_{\text {stato }}} \text { if } W \tan \theta<2 H_{\text {stato }} \\
\frac{\sqrt{2 H_{\text {stato }} W \tan \theta}}{2 H+(\mathcal{R}-1) \sqrt{2 H_{\text {stato }} W \tan \theta}} \text { if } 2 H_{\text {stato }}<W \tan \theta<H^{2} /\left(2 H_{\text {stato }}\right) \\
\frac{1}{\mathcal{R}+1} \text { else }
\end{array}\right.
$$

with

$$
\mathcal{A}=\frac{\alpha N_{\text {tot }} W N}{2 S \mathcal{D}} .
$$

The different conditions in Equation (19) stand for cases where statoliths are totally absent of the left side, or totally covering the right side (see Figure 7). Once the geometry of the cell and of the statoliths pile are fixed, the predicted gravitropic response depends on two dimensionless parameters: the parameter $\mathcal{R}$, which characterized the strength of the statolith-PIN coupling, and the parameter $\mathcal{A}$, which represents the ratio between the conductance of all PINs, $\alpha N_{\text {tot }}$, and the conductance of all efflux carriers, $2 S \mathcal{D} / W$ [see the expression (9) of $\mathcal{D}$ ], multiplied by the number of cells $N$. The maximal amplitude of the gravitropic response is obtained at $90^{\circ}$ and is equal to $(1 / 2) \mathcal{A}(\mathcal{R}-$ $1) /(\mathcal{R}+1)$. For large $\mathcal{R}$, i.e., when PINs localize predominantly when statoliths are present, the gravitropic response is thus mainly fixed by $\mathcal{A}$. In Figure 7, we present the experimental measurements of the "sine-law" obtained by Chauvet et al. (2016) on wheat coleoptiles, together with the best fit of the data using a least-square method. Reasonable agreement between theory and experiments is obtained with $\mathcal{R} \simeq 25$ (or larger values as the shape converges in this case) and $\mathcal{A} \simeq 1$. It is interesting to note that within our position-sensor framework, the predicted steady gravitropic response is not a simple "sinelaw," but rather a piece-wise curve with an overall concave shape. This law is also not universal and can be affected by several anatomical and physiological properties, such as the geometry of the cell $H / W$, the amount of statoliths $H_{\text {stato }} / W$, or the molecular signaling machinery (embedded in the parameters $\mathcal{R}$ and $\mathcal{A}$ ).
( $\sim 2 \mathrm{~min}$ ) but shorter than the growth timescale (hours). It thus reflects a temporal process in the gravitropic signaling pathway that remains to be identified. We address below this question in the framework of the model.

The set of Equations (7)-(10) give a complete description of the transient gravitropic response (we assume that sedimentation of statoliths is fast enough that the surfaces in Equation $10 \mathrm{can}$ be computed from their equilibrium values-see Appendix B). Two different typical times control the dynamics: $\tau_{\mathrm{aux}}$, describing the transport of auxin across the tissue of length $2 R$, and $\tau_{\mathrm{PIN}}$, describing the dynamics of PIN at molecular scale. The time scale associated to auxin transport $\tau_{\text {aux }}$ can be estimated using Equation (7) for a constant coefficient of diffusion $\mathcal{D}$ and transport velocity $v$ (i.e., PIN distribution). In this case, relaxation toward the stationary profile (Equation 12) is exponential and occurs on a time scale set by the inverse of the shortest nonvanishing eigen-mode of Equation (7) (Mohsen and Baluch, 1983):

$$
\tau_{\text {aux }}=\frac{1}{\pi^{2}+\left(\mathrm{Pe}^{2} / 4\right)} \times \frac{(2 R)^{2}}{\mathcal{D}}=\frac{1}{\pi^{2}+\left(\mathrm{Pe}^{2} / 4\right)} \times \frac{(2 R) \mathrm{Pe}}{v}
$$

Estimating the Peclet number from the steady gravitropic response $\left(\mathrm{Pe}=2 \tilde{\Delta}^{\text {steady }} \approx 1\right.$, see Figure 7$)$, and the auxin transport speed $v$ from measurements of the speed of auxin pulses in plant tissues $(v \simeq 3 \mu \mathrm{m} / \mathrm{s}$, Goldsmith, 1977; Rashotte et al., 2003), gives $\tau_{\text {aux }} \simeq 30 \mathrm{~s}$ (taking $2 R \sim 1 \mathrm{~mm}$ ). This is much shorter than the memory time $\tau_{\text {memory }} \sim 15 \mathrm{~min}$ evidenced in dose-response experiments, and even shorter than the statoliths sedimentation time. This suggests that the dynamics of the gravitropic response is controlled by $\tau_{\mathrm{PIN}}$, rather than by the auxin diffusion time $\tau_{\text {aux }}$. 


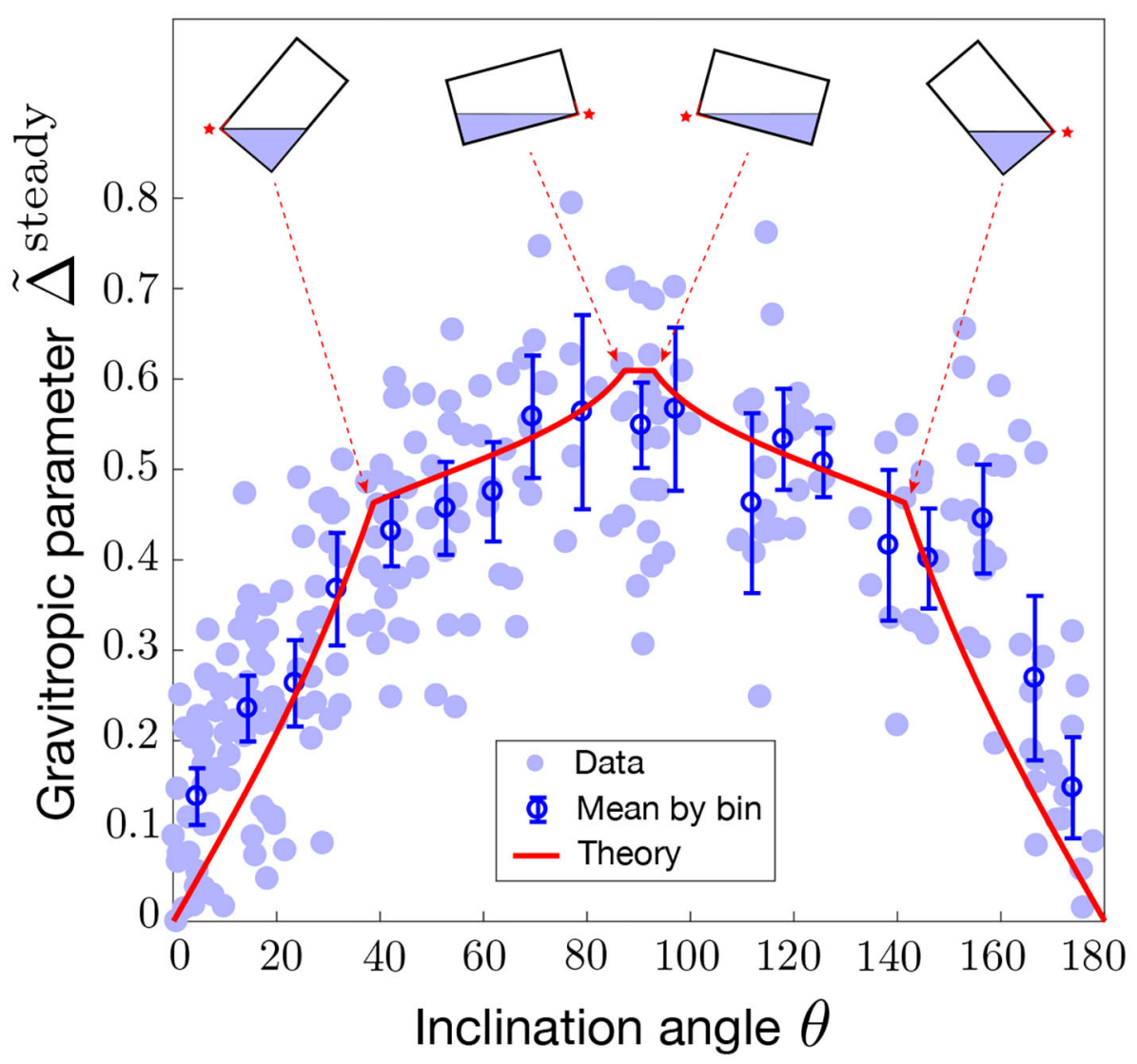

FIGURE 7 | Modified sine-law $\tilde{\Delta}^{\text {steady }}$ as a function of the inclination angle $\theta$. Comparison between the model prediction for $\mathcal{P} \ll 1$ (limiting pool) and L-binding (Equation 19 with $\mathcal{R}=25$ and $\mathcal{A}=1.3$, red line) and experiments on wheat coleoptiles (symbols, Chauvet et al., 2016). Geometrical parameters used in the model for the statocyte are $H_{\text {stato }}=4 d, W=10 d, H=25 d$ where $d$ stands for the diameter of a statolith. Error bars are the mean value of the data by binning the [0,180] interval into 20 boxes.

The time scale $\tau_{\text {PIN }}$ is set by the slowest characteristic time of the system (Equation 10) describing PIN dynamics. From the eigenvalues of this linear system, two timescales are obtained, which are solutions of:

$$
\begin{array}{r}
\tau^{-2}-\left(k_{\mathrm{off}, 0}+k_{\mathrm{off}, 1}+\left[k_{\mathrm{on}, 0} S_{0}\right.\right. \\
\left.\left.+k_{\mathrm{on}, 1} S_{1}\right] / W S\right) \tau^{-1}+\left(k_{\mathrm{off}, 0}\right. \\
\left.+k_{\mathrm{on}, 0} S_{0} / W S\right)\left(k_{\mathrm{off}, 1}+k_{\mathrm{on}, 1} S_{1} / W S\right) \\
-k_{\mathrm{on}, 0} k_{\mathrm{on}, 1} S_{0} S_{1} / W^{2} S^{2}=0 .
\end{array}
$$

In the limiting pool case ( $\left.W S k_{\mathrm{off}, \mathrm{i}} / k_{\mathrm{on}, \mathrm{i}} S_{i} \ll 1\right)$, the two solutions are:

$$
\begin{aligned}
\tau_{1} & =\frac{W S}{k_{\mathrm{on}, 0} S_{0}+k_{\mathrm{on}, 1} S_{1}} \quad \text { and } \\
\tau_{2} & =\frac{k_{\mathrm{on}, 0} S_{0}+k_{\mathrm{on}, 1} S_{1}}{k_{\mathrm{off}, 0} k_{\mathrm{on}, 1} S_{1}+k_{\mathrm{off}, 1} k_{\mathrm{on}, 0} S_{0}},
\end{aligned}
$$

with $\tau_{1} \ll \tau_{2}$. Therefore, the slowest timescale of the gravitropic

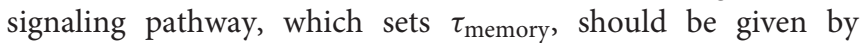
$\tau_{\text {PIN }}=\tau_{2}$. Note that when preferential attachment in region with statoliths is achieved via a strongly increased attachment rate $\left(k_{\mathrm{on}, 1} \gg k_{\mathrm{on}, 0}\right)$, and not by change of detachment rate $\left(k_{\mathrm{off}, 0} \simeq\right.$ $\left.k_{\mathrm{off}, 1}\right)$, then $\tau_{\mathrm{PIN}}=k_{\mathrm{off}}^{-1}$. Conversely, if it is achieved by decreased detachment rate $\left(k_{\mathrm{off}, 0} \gg k_{\mathrm{off}, 1}\right)$ and not by change of attachment rate $\left(k_{\mathrm{on}, 0} \simeq k_{\mathrm{on}, 1}\right)$, then $\tau_{\mathrm{PIN}}=k_{\mathrm{off}, 0}^{-1}\left(1+S_{1} / S_{0}\right) \simeq k_{\mathrm{off}, 0}^{-1}$. Remarkably, in this last case, the equilibration time of PIN is not controlled by the slowest rate of detachment $\left(k_{\mathrm{off}, 1}\right)$, but by the fastest one $\left(k_{\mathrm{off}, 0}\right)$, due to the limiting pool.

To check these predictions, we solve the model for a transient gravitropic stimulus that reproduces the protocol of Chauvet et al. (2019). The inclination $\theta$ is set to $45^{\circ}$ for a transient time $\Delta T$ and then put back to zero, the gravitropic response being defined as the maximal auxin gradient reached during the dynamics: $\tilde{\Delta}^{\text {trans }}=\operatorname{Max}\left[\frac{c_{\text {bottom }}(t)-c_{\text {top }}(t)}{2 c_{\text {mean }}}\right]$. Figure 8A compares the experimental data of Chauvet et al. (2019) with the prediction of the model for $\tau_{\text {aux }} / \tau_{\text {PIN }} \ll 1$, using the parameters $\mathcal{R}$ and $\mathcal{A}$ already fixed by the steady response (see Figure 7). Good agreement is obtained using $\tau_{\mathrm{PIN}}=13 \mathrm{~min}$ as the only fitting parameter. This result shows that $\tau_{\mathrm{PIN}}$ is playing the role of the memory time evidenced by the experiments of Chauvet et al. (2019). If $\Delta T \ll \tau_{\mathrm{PIN}}$, PIN transporters have not enough 

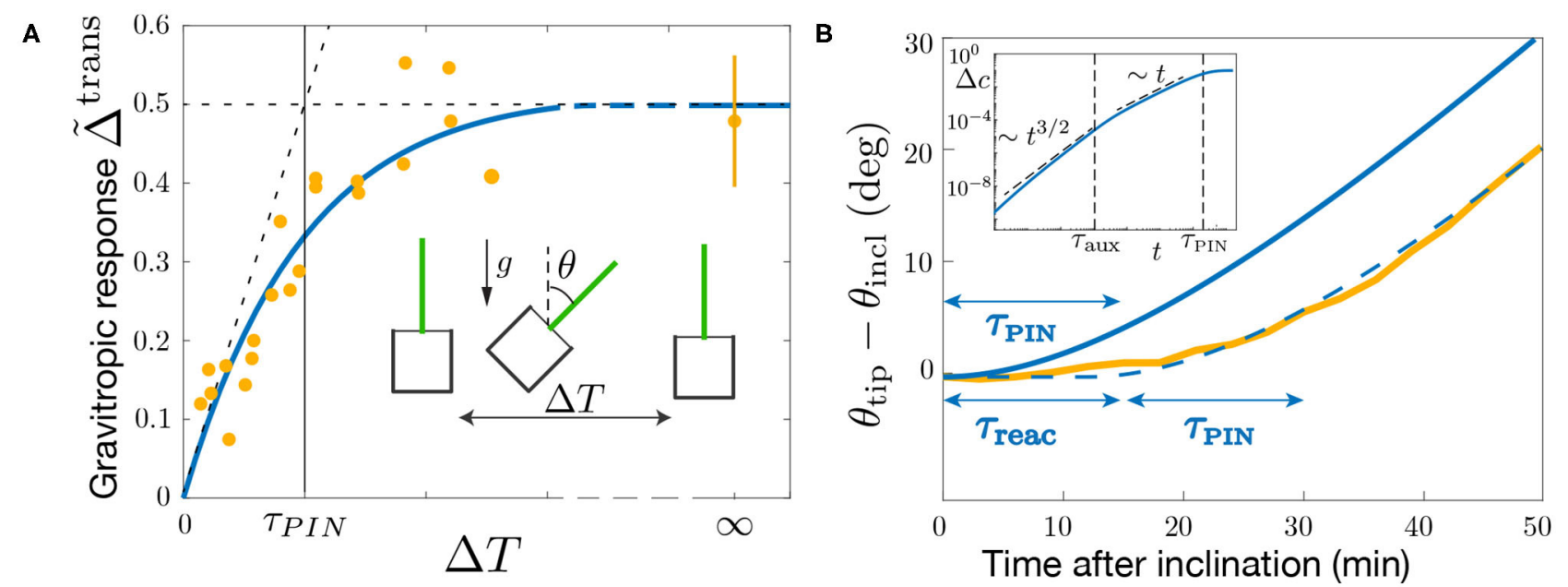

FIGURE 8 | Gravitropic response to a transient inclination (dose-response like protocol). (A) Maximal gravitropic response reached during the dynamics as function of the inclination time $\Delta T$ for $\theta=45^{\circ}$. The blue solid line is the model prediction using $\tau_{\mathrm{PIN}}=13 \min \left(\tau_{\mathrm{aux}} / \tau_{\mathrm{PIN}}=1 / 2,000\right.$ and $\tau_{1} / \tau_{\mathrm{PIN}}=0$, other parameters are fixed as in Figure 7). Note that the curve is not sensitive to the exact value of $\tau_{\mathrm{aux}} / \tau_{\mathrm{PIN}}$ and $\tau_{1} / \tau_{\mathrm{PIN}}$ as long as these values are small. Note also that the curve does not depend on $\mathcal{P}$ in the limiting pool regime $\mathcal{P} \ll 1$ considered. Symbols correspond to the results of Chauvet et al. (2019) obtained under normal Earth gravity (1g) and hypergravity condition $(3 \mathrm{~g})$. (B) Evolution of the tip angle after an inclination $\theta=50^{\circ}$ predicted by the model with the same parameters (blue solid line) and in the experiments of Chauvet et al. (2016) (orange thick line). The predicted model must be shifted by a constant time $\tau_{\text {reac }}=13$ min to match the experimental curve (dashed blue line). Inset: early time behavior of the gravitropic response predicted by the model in log-log scale.

time to rearrange before the end of the stimulus, and the response is weak. Conversely if $\Delta T \gg \tau_{\mathrm{PIN}}$, PINs have time to rearrange and reach their steady repartition before the end of the stimulus, and the response is maximal, similar to the one of a permanent stimulus.

We finish our analysis by investigating the full temporal dynamics of the gravitropic response after a sudden inclination $\theta=50^{\circ}$. Figure 8B presents the time evolution of the shoot curvature $\mathcal{C}(t)$ (or similarly $\theta_{\text {tip }}(t)$ for small curvatures) predicted by the model for $\tau_{\mathrm{PIN}}=13 \mathrm{~min}$ (blue curve), together with the experimental data of Chauvet et al. (2016) (yellow curve). The time scale of the curvature change $\tau_{\text {PIN }}$ is well captured by the model. However, to match the experiments, the model has to be shifted in time by a constant time $\tau_{\text {reac }} \approx 13 \mathrm{~min}$. Such delay or reaction time of the gravitropic response after the stimulus was already noticed by Chauvet et al. (2019), but does not seem to be captured by the model. Actually, a careful analysis of the temporal behavior of the model (assuming $\tau_{1} \ll \tau_{\text {aux }} \ll \tau_{\text {PIN }}=\tau_{2}$ ) shows that the auxin gradient at early times increases as $\Delta c \sim t^{3 / 2}$ (so that $\mathcal{C} \propto \int_{0}^{t} \Delta c \mathrm{~d} t \propto t^{5 / 2}$ ) as long as $t<\tau_{\text {aux }}$, before varying as $\Delta c \sim t$ for $\tau_{\text {aux }}<t<\tau_{\text {PIN }}$ (Inset of Figure 8B). These scaling laws are thus not compatible with a very flat initial response.

\section{DISCUSSION}

In this paper, we have derived a multi-scale model of plant gravitropism which links the different steps of the gravitropic signaling pathway: (i) the initial intracellular perception of gravity by statoliths, (ii) the transduction of this physical signal into a biochemical signal through the reorganization of PINs at the membrane of statocytes, (iii) the intercellular signal transmission via auxin transport, and (iv) asymmetric organ growth. The model is based on several simplifications and assumptions: we only consider a one-dimensional model consisting in an array of identical cells where transport occurs in the transverse direction only, and neglect auxin metabolism using a continuum approach. These assumptions are summarized in Table 3 together with possible improvements and extensions. However, the main originality of the model lies in the mechanistic link we propose between the statoliths position and the dynamics of PINs, based on the recent position-sensor hypothesis (Pouliquen et al., 2017). This basic assumption enables us to recover several major features of the gravitropic response of plants.

\subsection{A New Interpretation of the Sine-Law of Plant Gravitropism}

The first main result concerns the steady gravitropic response to a permanent gravity stimulus, $\tilde{\Delta}^{\text {steady }}(\theta)$. For many plants, this response takes the form of an inclination-dependent law with a sine-like shape, called for this reason the sine-law (Sachs, 1887; Larsen, 1969; Iino et al., 1996; Galland, 2002; Dumais, 2013). This sine-law has long been interpreted in terms of a force sensor mechanism, for the projected weight of the statoliths on the lateral surface of the cell varies with the sine of the inclination angle (Audus, 1969; Barlow, 1995). However, recent experiments showing that the response is independent of the gravity intensity have dismissed this forcesensing hypothesis, calling for a new interpretation of the sine law (Chauvet et al., 2016; Pouliquen et al., 2017). A key result of the model is that it predicts an inclination-dependent steady 
gravitropic response $\tilde{\Delta}^{\text {steady }}(\theta)$ without invoking a force-based mechanism. In the model, the initial gravitropic stimulus is the statoliths position at the cell membrane, not their weight. Since statoliths behave on long time like a liquid (Bérut et al., 2018), their position in steady state is a purely geometrical cue, which depends only on the cell inclination. As a result, the steady response predicted by the model depends on the inclination but not on the gravity intensity, in agreement with the observations.

In the model, the actual shape of the gravitropic response $\tilde{\Delta}^{\operatorname{steady}}(\theta)$ is never a pure sine law. It depends on several parameters, related either to geometric factors, such as the aspect ratios of the statocytes and of the sedimented statoliths pile, or to molecular processes: intensity of coupling between statoliths position and PINs through parameter $\mathcal{R}$; ratio of endocytosis to exocytosis through parameter $\mathcal{P}$; ratio between the conductance of PIN carriers to the total conductance of auxin transporters through parameter $\mathcal{A}$. For elongated cells and shallow statoliths piles such as those of wheat coleoptile statocytes, the shape of the response tends to be sine-like only in the case of a strong coupling between the statoliths and PINs $(\mathcal{R} \gg 1)$, and for a number of PINs conserved along the cell membrane (limiting pool regime, $\mathcal{P} \ll 1$ ). This latter assumption is common in models of auxin transport (Runions et al., 2014; Retzer et al., 2019), while the strong coupling assumption is compatible with the large asymmetry in PINs localization observed upon gravity stimulation (Friml et al., 2002; Harrison and Masson, 2008; Kleine-Vehn et al., 2010). Interestingly, although gravity is absent from the model, the gravitropic response depends on the amount of statoliths through the geometrical aspect ratio of the statoliths pile $H_{0} / W$. The model could thus account for previous experiments using starch-less and starch-excess mutants, which showed a modified gravitropic response when the number of statoliths is changed (Kiss et al., 1997; Vitha et al., 2007; Pouliquen et al., 2017). Finally, it is worth noting that the model assumes that the statoliths form a static pile at the bottom of the cell, while statoliths actually exhibit a dynamic and random agitation due to the interaction with the cytoskeleton (Sack et al., 1986; Saito et al., 2005; Nakamura et al., 2011; Bérut et al., 2018). We might expect this agitation to reduce the averaged contact time between the statoliths and the cell membrane, thereby decreasing the coupling between statoliths and PINs. It would be interesting to extend the model in order to incorporate such effect of agitation on the gravitropic response. The model could then be compared with the behavior of agravitropic mutants in Arabidopsis thaliana like $\operatorname{sgr} 9$, whose weaker response is likely associated to an abnormally strong agitation of the statoliths (Nakamura et al., 2011).

Overall, our model suggests that the classical sine-law of plant gravitropism might not be universal, as its shape and amplitude could depend on several anatomical and physiological parameters. Full measurements of the gravitropic response of plants over a wide range of inclination are scarce and mostly performed on shoot coleoptiles. It would be interesting to perform systematic measurements of the sine-law on other plant organs (root, stem), to see if the shape of the sine-law is affected by different statocyte geometries and tissue organization.

\subsection{The Gravity-Independent Memory Process in Dose-Response Laws Is Likely Associated to PIN Dynamics}

The second main result of our study concerns the gravitropic response to a transient gravity stimulus. For a sudden inclination applied at time $t=0$, the model predicts that the response reaches the steady response $\tilde{\Delta}^{\text {steady }}(\theta)$ only after a time large compared to a "memory" timescale $\tau_{\text {memory }}$, corresponding to the slowest timescale introduced in the model. Therefore, when a stimulus is applied only during a transient time $\Delta T<$ $\tau_{\text {memory, a weaker gravitropic response is predicted, following }}$ a dose-response like law. In the model, the memory time is not associated with the sediment time of the statoliths, which is assumed to be much shorter than the other timescales of the gravitropic signaling pathway (a valid assumption for a gravity intensity like Earth gravity). Our model is therefore compatible with the recent experiments of Chauvet et al. (2019) performed on wheat coleoptiles, which show a doselike behavior of the gravitropic response with a memory time $\tau_{\text {memory }}$ independent of gravity. The model also provides the explicit origin of this memory process, which was postulated in Chauvet et al. (2019). In the model, two different processes can lead to the temporal filtration of the initial signal associated with statoliths position: auxin transport across the tissue and the dynamics of PIN turnover at the molecular scale. Our study suggests that the limiting process is actually controlled by PIN dynamics $\left(\tau_{\text {memory }}=\tau_{\text {PIN }}\right.$ ), auxin transport being too fast to account for the memory time measured experimentally ( 10-20 min). Interestingly, visualization of the PIN3 auxin efflux carrier in root columella cells after a sudden change in the gravity vector indicates a time scale of about 10 min for complete relocation (Friml et al., 2002), a duration very close to the memory time measured by Chauvet et al. (2019).

Although our model successfully captures the existence and origin of a gravity-independent memory process in the signaling pathway, it is not able to describe the delay time $\tau_{\text {reaction }} \sim 10 \mathrm{~min}$ observed between the application of the stimulus and the first gravitropic response (Chauvet et al., 2019). This delay may have different origins: the time needed to reorganize the cytoskeleton implied in the transport of PIN carriers, the time needed by a PIN to go from the pool toward the plasma membrane, or the time of incorporation of a PIN into the membrane. It is worth noting that a similar timescale of about $10 \mathrm{~min}$ was identified in the gravity-sensing columella cells for the internalization of PIN3 from the plasma membrane into vesicles (Kleine-Vehn et al., 2010). Further experiments combining a transient stimulus with pharmacological and genetic approaches would be needed to confirm the key role of the PIN turnover timescale in the gravitropic response. Besides, it would be interesting to see what is the influence of this hierarchy of time-scales on the response of a plant to oscillatory stimulus (e.g., wind) or on the dynamic 
competition between gravitropism and proprioception (Bastien et al., 2013).

\subsection{Back to the Statoliths/PIN Coupling Assumption}

We conclude by discussing the possible origin of the coupling between statoliths and PINs, which is at the core of our model. Although the respective roles of statoliths and PIN auxin transporters in plant gravitropism are well established, the link between the two is still not clear (see Nakamura et al., 2019b for a recent review of the possible molecular actors involved). In our model, we have used a very general hypothesis for this coupling based on the recent finding that the relevant gravitropic stimulus is the statoliths position inside the gravisensing cells (Chauvet et al., 2016; Pouliquen et al., 2017; Bérut et al., 2018). We have postulated that statoliths in contact with the cell membrane bias the exocytosis and endocytosis rate of PIN recycling, therefore inducing an asymmetry of PIN distribution when statoliths reposition in response to plant inclination. Our results suggest that this interaction between PINs and statoliths is strong, as large values of the parameter $\mathcal{R}$ are needed to match the experimental gravitropic response. This interaction between statoliths and PINs could involve a complex molecular pathway, such as TOC-protein (Strohm et al., 2014) or PID/WAG mediated phosphorylation (Rakusová et al., 2011; Grones et al., 2018), that remains to be unveiled. However, more direct mechanisms of interaction could occur. For example, statoliths could modify PIN vesicle-mediated transportation to the membrane by modifying the architecture of the actin cytoskeleton. Another possibility would be that internalization of PINs from the membrane is reduced by the presence of statoliths, for example by simple steric effects. Indeed, direct visualizations reveal a length scale of $\sim 1 \mu \mathrm{m}$ for the endosome formation, which is not far from statolith size (Kleine-Vehn et al., 2010). Interestingly, such direct interaction of statoliths and the cytoskeleton machinery was put forward as an explanation of gravitropism in rhizoids and protonemata, such as the single-cell alga Chara (Sievers et al., 1996). A last possibility could be that PINs cluster in regions where there is no statolith, while they do not cluster when statoliths are present. Indeed, for a conserved number of PIN proteins, clustering reduces the efflux efficiency, as this diffusion process

\section{REFERENCES}

Audus, L. (1969). "Geotropism," in The Physiology of Plant Growth and Development, ed M. B.Wilkins (London: McGraw-Hill), 203-241.

Band, L. R., Wells, D. M., Larrieu, A., Sun, J., Middleton, A. M., French, A. P., et al. (2012). Root gravitropism is regulated by a transient lateral auxin gradient controlled by a tipping-point mechanism. Proc. Natl. Acad. Sci. U.S.A. 109:4668. doi: 10.1073/pnas.1201498109

Barlow, P. W. (1995). Gravity perception in plants: a multiplicity of systems derived by evolution? Plant Cell Environ. 18, 951-962. doi: 10.1111/j.1365-3040.1995.tb00606.x

Bastien, R., Bohr, T., Moulia, B., and Douady, S. (2013). Unifying model of shoot gravitropism reveals proprioception as a central feature of scales not linearly but as the square-root of the number of carriers (Bénichou and Voituriez, 2014; Valet et al., 2019). Such a clustering has been highlighted by Kleine-Vehn et al. (2011), but, to our knowledge, no comparison has been done between regions of the membrane in contact or not in contact with statoliths.

Finally, we have assumed here that statoliths influence auxin transport through PIN trafficking only. Another possibility would be that statoliths change the PIN conductance to auxin without modifying their spatial distribution, for instance by interfering with D6PKs-induced PINs phosphorylation (Zourelidou et al., 2014; Nakamura et al., 2019a). In our model, this mechanism could be described by writing a conductance $\alpha$ that depends on the presence or not of the statoliths in Equations (8-9). In any cases, further studies are needed to discriminate between these molecular mechanisms.

\section{DATA AVAILABILITY STATEMENT}

Publicly available datasets were analyzed in this study. This data can be found at Chauvet et al. (2019).

\section{AUTHOR CONTRIBUTIONS}

NL, OP, and YF designed the research and wrote the paper. NL built and analyzed the model with inputs from OP and YF. All authors contributed to the article and approved the submitted version.

\section{FUNDING}

This work was supported by the European Research Council (ERC) under the European Union's Horizon 2020 research and innovation programme [grant agreement ( $\left.\mathrm{N}^{\circ} 647384\right)$ ].

\section{ACKNOWLEDGMENTS}

The authors would like to thank Valérie Legué, Félix Hartmann, and Bruno Moulia for insightful comments and a critical reading of the manuscript, as well as for the many stimulating discussions we have continually had on this subject. posture control in plants. Proc. Natl. Acad. Sci. U.S.A. 110, 755-760. doi: 10.1073/pnas.1214301109

Bastien, R., Douady, S., and Moulia, B. (2014). A unifying modeling of plant shoot gravitropism with an explicit account of the effects of growth. Front. Plant Sci. 5:136. doi: 10.3389/fpls.2014.00136

Bénichou, O., and Voituriez, R. (2014). From first-passage times of random walks in confinement to geometry-controlled kinetics. Phys. Rep. 539, 225-284. doi: 10.1016/j.physrep.2014. 02.003

Bérut, A., Chauvet, H., Legué, V., Moulia, B., Pouliquen, O., and Forterre, Y. (2018). Gravisensors in plant cells behave like an active granular liquid. Proc. Natl. Acad. Sci. U.S.A. 115:5123. doi: 10.1073/pnas.18018 95115 
Chauvet, H., Moulia, B., Legué, V., Forterre, Y., and Pouliquen, O. (2019). Revealing the hierarchy of processes and time-scales that control the tropic response of shoots to gravi-stimulations. J. Exp. Bot. 70, 1955-1967. doi: $10.1093 /$ jxb/erz027

Chauvet, H., Pouliquen, O., Forterre, Y., Legué, V., and Moulia, B. (2016). Inclination not force is sensed by plants during shoot gravitropism. Sci. Rep. 6:35431. doi: 10.1038/srep35431

Chelakkot, R., and Mahadevan, L. (2017). On the growth and form of shoots. J. R. Soc. Interface 14:20170001. doi: 10.1098/rsif.2017.0001

Darwin, C., and Darwin, S. F. (1880). The Power of Movements in Plants. London: John Murray. doi: 10.5962/bhl.title.102319

Dumais, J. (2013). Beyond the sine law of plant gravitropism. Proc. Natl. Acad. Sci. U.S.A. 110, 391-392. doi: 10.1073/pnas.1219974110

Dyson, R. J., Vizcay-Barrena, G., Band, L. R., Fernandes, A. N., French, A. P., Fozard, J. A., et al. (2014). Mechanical modelling quantifies the functional importance of outer tissue layers during root elongation and bending. New Phytol. 202, 1212-1222. doi: 10.1111/nph.12764

Fendrych, M., Akhmanova, M., Merrin, J., Glanc, M., Hagihara, S., Takahashi, K., et al. (2018). Rapid and reversible root growth inhibition by TIR1 auxin signalling. Nat. Plants 4, 453-459. doi: 10.1038/s41477-0180190-1

Friml, J., Wiśniewska, J., Benková, E., Mendgen, K., and Palme, K. (2002). Lateral relocation of auxin efflux regulator PIN3 mediates tropism in Arabidopsis. Nature 415, 806-809. doi: 10.1038/415806a

Galland, P. (2002). Tropisms of avena coleoptiles: sine law for gravitropism, exponential law for photogravitropic equilibrium. Planta 215, 779-784. doi: 10.1007/s00425-002-0813-6

Galston, A. W., and Hand, M. E. (1949). Studies on the physiology of light action; auxin and the light inhibition of growth. Am. J. Bot. 36, 85-94. doi: 10.1002/j.1537-2197.1949.tb05235.x

Goldsmith, M. H. M. (1977). The polar transport of auxin. Annu. Rev. Plant Physiol. 28, 439-478. doi: 10.1146/annurev.pp.28.060177.002255

Grieneisen, V. A., Scheres, B., Hogeweg, P., and M Marée, A. F. (2012). Morphogengineering roots: comparing mechanisms of morphogen gradient formation. BMC Syst. Biol. 6:37. doi: 10.1186/1752-0509-6-37

Grones, P., Abas, M., Hajný, J., Jones, A., Waidmann, S., Kleine-Vehn, J., et al. (2018). PID/WAG-mediated phosphorylation of the arabidopsis PIN3 auxin transporter mediates polarity switches during gravitropism. Sci. Rep. 8, 1-11. doi: 10.1038/s41598-018-28188-1

Harrison, B. R., and Masson, P. H. (2008). ARL2, ARG1 and PIN3 define a gravity signal transduction pathway in root statocytes. Plant J. 53, 380-392. doi: 10.1111/j.1365-313X.2007.03351.x

Hopkins,W. G., and Hüner,N. P. (2009). Introduction to Plant Physiology, 4th Edn. Hoboken, NJ: John Wiley \& Sons.

Iino, M., Tarui, Y., and Uematsu, C. (1996). Gravitropism of maize and rice coleoptiles: dependence on the stimulation angle. Plant Cell Environ. 19, 1160-1168. doi: 10.1111/j.1365-3040.1996.tb00431.x

Kiss, J. Z., Guisinger, M. M., Miller, A. J., and Stackhouse, K. S. (1997). Reduced gravitropism in hypocotyls of starch-deficient mutants of arabidopsis. Plant Cell Physiol. 38, 518-525. doi: 10.1093/oxfordjournals.pcp.a029199

Kleine-Vehn, J., Ding, Z., Jones, A. R., Tasaka, M., Morita, M. T., and Friml, J. (2010). Gravity-induced pin transcytosis for polarization of auxin fluxes in gravity-sensing root cells. Proc. Natl. Acad. Sci. U.S.A. 107, 22344-22349. doi: $10.1073 /$ pnas. 1013145107

Kleine-Vehn, J., and Friml, J. (2008). Polar targeting and endocytic recycling in auxin-dependent plant development. Annu. Rev. Cell Dev. Biol. 24, 447-473. doi: 10.1146/annurev.cellbio.24.110707.175254

Kleine-Vehn, J., Wabnik, K., Martiniére, A., Łangowski, Ł., Willig, K., Naramoto, S., et al. (2011). Recycling, clustering, and endocytosis jointly maintain pin auxin carrier polarity at the plasma membrane. Mol. Syst. Biol. 7:540. doi: $10.1038 / \mathrm{msb} .2011 .72$

Kramer, E. M., and Ackelsberg, E. M. (2015). Auxin metabolism rates and implications for plant development. Front. Plant Sci. 6:150. doi: $10.3389 /$ fpls.2015.00150

Kramer, E. M., Frazer, N. L., and Baskin, T. I. (2007). Measurement of diffusion within the cell wall in living roots of Arabidopsis thaliana. J. Exp. Bot. 58, 3005-3015. doi: 10.1093/jxb/erm 155
Krecek, P., Skupa, P., Libus, J., Naramoto, S., Tejos, R., Friml, J., et al. (2009). The pin-formed (PIN) protein family of auxin transporters. Genome Biol. 10:249. doi: $10.1186 / \mathrm{gb}-2009-10-12-249$

Kutschera, U., and Niklas, K. (2007). The epidermal-growth-control theory of stem elongation: an old and a new perspective. J. Plant Physiol. 164, 1395-1409. doi: 10.1016/j.jplph.2007.08.002

Larsen, P. (1969). The optimum angle of geotropic stimulation and its relation to the starch statolith hypothesis. Physiol. Plant. 22, 469-488. doi: 10.1111/j.1399-3054.1969.tb07401.x

Meroz, Y., Bastien, R., and Mahadevan, L. (2019). Spatio-temporal integration in plant tropisms. J. R. Soc. Interface 16:20190038. doi: 10.1098/rsif.2019.0038

Mohsen, M. F. N., and Baluch, M. H. (1983). An analytical solution of the diffusionconvection equation over a finite domain. Appl. Math. Model. 7, 285-287. doi: 10.1016/0307-904X(83)90084-7

Morita, M. T. (2010). Directional gravity sensing in gravitropism. Annu. Rev. Plant Biol. 61, 705-720. doi: 10.1146/annurev.arplant.043008.092042

Moulia, B., and Fournier, M. (2009). The power and control of gravitropic movements in plants: a biomechanical and systems biology view. J. Exp. Bot. 60, 461-486. doi: 10.1093/jxb/ern341

Moulton, D. E., Oliveri, H., and Goriely, A. (2020). Multiscale integration of environmental stimuli in plant tropism produces complex behaviors. Proc. Natl. Acad. Sci. U.S.A. 117, 32226-32237. doi: 10.1073/pnas.2016025117

Nakamura, M., Nishimura, T., and Morita, M. T. (2019a). Bridging the gap between amyloplasts and directional auxin transport in plant gravitropism. Curr. Opin. Plant Biol. 52, 54-60. doi: 10.1016/j.pbi.2019.07.005

Nakamura, M., Nishimura, T., and Morita, M. T. (2019b). Gravity sensing and signal conversion in plant gravitropism. J. Exp. Bot. 70, 3495-3506. doi: $10.1093 /$ jxb/erz158

Nakamura, M., Toyota, M., Tasaka, M., and Morita, M. T. (2011). An Arabidopsis E3 ligase, SHOOT GRAVITROPISM9, modulates the interaction between statoliths and F-actin in gravity sensing. Plant Cell 23, 1830-1848. doi: 10.1105/tpc.110.079442

Pouliquen, O., Forterre, Y., Bérut, A., Chauvet, H., Bizet, F., Legue, V., et al. (2017). A new scenario for gravity detection in plants: the position sensor hypothesis. Phys. Biol. 14:035005. doi: 10.1088/1478-3975/aa6876

Rakusová, H., Gallego-Bartolomé, J., Vanstraelen, M., Robert, H. S., Alabadí, D., Blázquez, M. A., et al. (2011). Polarization of PIN3-dependent auxin transport for hypocotyl gravitropic response in arabidopsis thaliana. Plant J. 67, 817-826. doi: 10.1111/j.1365-313X.2011.04636.x

Rashotte, A. M., Poupart, J., Waddell, C. S., and Muday, G. K. (2003). Transport of the two natural auxins, indole-3-butyric acid and indole-3-acetic acid, in arabidopsis. Plant Physiol. 133:761. doi: 10.1104/pp.103.022582

Retzer, K., Akhmanova, M., Konstantinova, N., Malínská, K., Leitner, J., Petrášek, J., et al. (2019). Brassinosteroid signaling delimits root gravitropism via sorting of the arabidopsis PIN2 auxin transporter. Nat. Commun. 10, 1-15. doi: 10.1038/s41467-019-13543-1

Runions, A., Smith, R. S., and Prusinkiewicz, P. (2014). "Computational models of auxin-driven development," in Auxin and Its Role in Plant Development, eds E. Zažímalová, J. Petrasek, and E. Benková (New York, NY: Springer), 315-357. doi: 10.1007/978-3-7091-1526-8_15

Sachs, J. (1887). Lectures on the Physiology of Plants. Oxford: Clarendon Press. doi: 10.5962/bhl.title.54852

Sack, F. D., Suyemoto, M. M., and Leopold, A. C. (1986). Amyloplast sedimentation and organelle saltation in living corn columella cells. Am. J. Bot. 73, 1692-1698. doi: 10.1002/j.1537-2197.1986.tb09700.x

Saito, C., Morita, M. T., Kato, T., and Tasaka, M. (2005). Amyloplasts and vacuolar membrane dynamics in the living graviperceptive cell of the arabidopsis inflorescence stem. Plant Cell 17, 548-558. doi: 10.1105/tpc.104. 026138

Sievers, A., Buchen, B., and Hodick, D. (1996). Gravity sensing in tip-growing cells. Trends Plant Sci. 1, 249-250. doi: 10.1016/1360-1385(96)10028-5

Silk, W. K. (1984). Quantitative descriptions of development. Annu. Rev. Plant Physiol. 35, 479-518. doi: 10.1146/annurev.pp.35.060184. 002403

Strohm, A. K., Barrett-Wilt, G. A., and Masson, P. H. (2014). A functional TOC complex contributes to gravity signal transduction in arabidopsis. Front. Plant Sci. 5:148. doi: 10.3389/fpls.2014.00148 
Toyota, M., and Gilroy, S. (2013). Gravitropism and mechanical signaling in plants. Am. J. Bot. 100, 111-125. doi: 10.3732/ajb.1200408

Valet, M., Pontani, L.-L., Voituriez, R., Wandersman, E., and Prevost, A. M. (2019). Diffusion through nanopores in connected lipid bilayer networks. Phys. Rev. Lett. 123:088101. doi: 10.1103/PhysRevLett.123.088101

Vitha, S., Yang, M., Sack, F. D., and Kiss, J. Z. (2007). Gravitropism in the starch excess mutant of arabidopsis thaliana. Am. J. Bot. 94, 590-598. doi: 10.3732/ajb.94.4.590

Zažímalová, E., Murphy, A. S., Yang, H., Hoyerová, K., and Hošek, P. (2010). Auxin transporters-why so many? Cold Spring Harb. Perspect. Biol. 2:a001552. doi: $10.1101 /$ cshperspect.a001552

Zourelidou, M., Absmanner, B., Weller, B., Barbosa, I. C., Willige, B. C., Fastner, A., et al. (2014). Auxin efflux by pin-formed proteins is activated by two different protein kinases, D6 protein kinase and pinoid. Elife 3:e02860. doi: 10.7554/eLife. 02860

Conflict of Interest: The authors declare that the research was conducted in the absence of any commercial or financial relationships that could be construed as a potential conflict of interest.

Copyright $(2021$ Levernier, Pouliquen and Forterre. This is an open-access article distributed under the terms of the Creative Commons Attribution License (CC BY).

The use, distribution or reproduction in other forums is permitted, provided the original author(s) and the copyright owner(s) are credited and that the original publication in this journal is cited, in accordance with accepted academic practice. No use, distribution or reproduction is permitted which does not comply with these terms. 


\section{APPENDIX A: CASE OF STATOLITHS ONLY PRESENT ON A RING}

Let be a central region between $l$ and $2 R-l$ with no statolith (and constant efflux rate $P_{0}$ ). We can redo the calculation leading to Equation (5) in this geometry. Clearly, equations will be similar in each region, with $v=0$ in the central one. We have to take care to the continuity of the flux at the junction of each regions. We can show that $J(l)=(P+\delta P) c\left(l^{-}\right)-P_{0} c\left(l^{+}\right)$and $J(2 R-l)=P_{0} c\left((2 R-l)^{-}\right)-P c\left((2 R-l)^{+}\right)$. At steady state, we thus get a jump of auxin concentration: $c\left(l^{+}\right)=(P+\delta P) / P_{0} c\left(l^{-}\right)$ and $c\left((2 R-l)^{+}\right)=P_{0} / P c\left((2 R-l)^{-}\right)$. In the limit of small Peclet number, we thus get $\Delta c / c=\left(P_{0} / P\right)(v l / \mathcal{D})$.

\section{APPENDIX B: EXPRESSION OF THE GEOMETRIC PARAMETERS AS A FUNCTION OF $\theta$}

We give here the value of the surfaces $S_{i}^{r, l}(\theta)$ that are necessary to compute the Peclet number as a function of the angle (see Equation 19). As statoliths are solid, the shaded area in Figure 3 is conserved and is thus equal to $H_{\text {stato }} W$. We note $\theta_{1}=$ $\arctan 2 H_{\text {stato }} / W$ the angle for which the horizontal level meets the lower left corner, and $\theta_{2}=\arctan H^{2} /\left(2 H_{\text {stato }} W\right)$ the one for which it meets the upper right one (we assume $H_{\text {stato }}<H / 2$ ). Elementary geometry calculations give:

$$
S_{0}^{l}=\left\{\begin{array}{l}
H-H_{\text {stato }}+\frac{W \tan \theta}{2} \text { if } \theta<\theta_{1} \\
H \text { else }
\end{array}\right.
$$

$$
\begin{aligned}
& S_{1}^{l}=\left\{\begin{array}{l}
H_{\text {stato }}-\frac{W \tan \theta}{2} \text { if } \theta<\theta_{1} \\
0 \text { else }
\end{array}\right. \\
& S_{0}^{r}= \begin{cases}H-H_{\text {stato }}-\frac{W \tan \theta}{2} & \text { if } \theta<\theta_{1} \\
H-\sqrt{2 H_{\text {stato }} W \tan \theta} & \text { if } \theta_{1}<\theta<\theta_{2} \\
0 \text { else } & \end{cases} \\
& S_{1}^{r}=\left\{\begin{array}{l}
H_{\text {stato }}+\frac{W \tan \theta}{2} \text { if } \theta<\theta_{1} \\
\sqrt{2 H_{\text {stato }} W \tan \theta} \text { if } \theta_{1}<\theta<\theta_{2} \\
H \text { else }
\end{array}\right.
\end{aligned}
$$

In the case of apical binding, this leads to

$$
\begin{gathered}
S_{0}=\left\{\begin{array}{l}
2\left(H-H_{\text {stato }}\right) \text { if } \theta<\theta_{1} \\
2 H-\sqrt{2 H_{\text {stato }} W \tan \theta} \text { if } \theta_{1}<\theta<\theta_{2} \\
H \text { else }
\end{array}\right. \\
S_{1}=\left\{\begin{array}{l}
2 H_{\text {stato }} \text { if } \theta<\theta_{1} \\
\sqrt{2 H_{\text {stato }} W \tan \theta} \text { if } \theta_{1}<\theta<\theta_{2} \\
H \text { else }
\end{array}\right.
\end{gathered}
$$

In the case of apical/basal/lateral binding, the expressions for the total surfaces are slightly modified due to attachment at apical and basal side. 